\title{
Coordination interindividuelle et performance en aviron
}

Apports d'une analyse conjointe du cours d'expérience des rameurs et de paramètres mécaniques

Interpersonal coordination and performance in rowing: contributions from a joint analysis of rowers' courses of experience and of mechanical parameters

Jacques Saury, Antoine Nordez et Carole Sève

\section{OpenEdition \\ Journals}

Édition électronique

URL : http://journals.openedition.org/activites/2369

DOI : 10.4000/activites.2369

ISSN : 1765-2723

Éditeur

ARPACT - Association Recherches et Pratiques sur les ACTivités

\section{Référence électronique}

Jacques Saury, Antoine Nordez et Carole Sève, « Coordination interindividuelle et performance en aviron », Activités [En ligne], 7-1 | avril 2010, mis en ligne le 15 avril 2010, consulté le 19 avril 2019. URL : http://journals.openedition.org/activites/2369 ; DOI : 10.4000/activites.2369

\section{(c) (i) $\Theta$}

Activités est mis à disposition selon les termes de la licence Creative Commons Attribution - Pas d'Utilisation Commerciale - Pas de Modification 4.0 International. 


\title{
Coordination interindividuelle et performance en aviron : apports d'une analyse conjointe du cours d'expérience des rameurs et de paramètres mécaniques
}

\author{
Jacques Saury \\ Laboratoire « Motricité, Interactions, Performance » (EA 4334), Université de Nantes \\ UFR STAPS, 25 bis, boulevard Guy Mollet, BP 72206, 44322 Nantes Cedex 3 \\ jacques.saury@univ-nantes.fr
}

Antoine Nordez

Laboratoire « Motricité, Interactions, Performance » (EA 4334), Université de Nantes antoine.nordez@univ-nantes.fr

\section{Carole Sève}

Laboratoire « Motricité, Interactions, Performance » (EA 4334), Université de Nantes carole.seve@univ-nantes.fr

\begin{abstract}
Interpersonal coordination and performance in rowing: contributions from a joint analysis of rowers' courses of experience and of mechanical parameters. The aim of this study was to analyse the phenomena associated with coordination between a crew of rowers during a race by confronting an analysis of the rowers' courses of experience and an analysis of correlative mechanical parameters of their activity during the race. It was designed (a) to acquire a better understanding of performance and to optimize the rowers' training, and (b) to explore the usefulness of a method that describes the processes of coordination at both significant and non-significant levels for the actors. Two female crews volunteered to participate in the study. Their activity was studied in situ during two time trials, relating to the theoretical and methodological framework of the course of action (Theureau, 2006). Mechanical measurements were collected, enabling a set of parameters on the rowers' performance and coordination to be calculated. The results are presented in two parts. The first describes the significant phenomena for the rowers related to their coordination. Three phenomena were highlighted: (a) a particular sensitivity to their state of coordination, (b) a recurring interpretation of their partners' activity, and (c) four typical ways of making mutual adjustments. In the second part, a case study analyses one specific moment in a race, experienced by the rowers as a malfunctioning of their coordination, by confronting analyses of the rowers' courses of experience and mechanical parameters. These findings are discussed at three levels, answering the empirical, methodological and practical aims of the study.
\end{abstract}

KEYWORDS

Sport, team performance, rowing, interpersonal coordination, course of experience, course of information. 


\section{1.- Introduction}

\section{1.- La coordination au sein des équipages d'aviron}

Les spécialités d'aviron en équipage concernent une pluralité de bateaux d'aviron, qui se distinguent en fonction de trois caractéristiques: (a) leur armement, qui définit deux catégories, dites respectivement « de pointe » (chaque rameur dispose d'un seul aviron) et « de couple » (chaque rameur dispose de deux avirons); (b) le nombre de rameurs (deux, quatre ou huit); et (c) la présence ou non d'un barreur dans l'équipage (équipier ne disposant pas d'aviron, chargé en particulier de diriger le bateau). La pratique de l'aviron, dans toutes ces spécialités, suppose une activité motrice cyclique de l'équipage fortement contrainte mécaniquement. Dans l'une des études les plus fréquemment citée à propos de la coordination entre rameurs, Wing et Woodburn (1995) ont avancé que les trois composantes essentielles d'une coordination efficace au sein d'un équipage d'aviron étaient: une cadence commune des coups d'aviron entre les rameurs, une synchronisation des différentes phases de chaque coup d'aviron (phase de propulsion dans l'eau et retour aérien), et une courbe force-temps similaire des coups d'aviron des différents rameurs.

Les facteurs sous-jacents de la performance collective des équipages d'aviron ont été appréhendés de différentes manières dans la littérature professionnelle, technique et scientifique du domaine.

Un examen des publications fédérales, manuels d'entraînement, et traités techniques, fait apparaître que deux questions majeures sont associées à l'optimisation de la performance collective en aviron dans le milieu de l'entraînement de haut niveau en France (e.g., Gossé, Carpentier, \& Avanzini, 2008), mais aussi plus largement dans les autres grandes nations d'aviron (e.g., Nolte, 2005). La première concerne la constitution des équipages, en fonction des qualités physiques, des caractéristiques biomécaniques et des compétences techniques individuelles des rameurs (Nilsen, 2005 ; Gossé et al., 2008 ). La deuxième question concerne les dimensions psychologiques liées à la performance collective des équipages : certains entraîneurs évoquent notamment les conditions permettant qu'une «dynamique collective » s'instaure au sein de ces équipages (Gossé et al., 2008), ou l'existence d'une « unité interne » au sein des équipages (Nilsen, 2005). Si la première question est bien documentée dans la littérature technique, la deuxième semble davantage relever d'une démarche intuitive et de l'expertise tacite des entraîneurs, capables de générer une « alchimie de l'effort collectif » (Gossé et al., 2008), sans que celle-ci soit nécessairement dénuée d'antagonismes interindividuels au sein d'un même équipage (Lenk, 1969; Nilsen, 2005). Cette observation concorde avec une conception partagée dans l'entraînement en aviron, selon laquelle la performance d'un équipage dépend avant tout des performances individuelles des rameurs et de la pertinence de l'agencement de ces derniers dans le bateau.

Les études scientifiques sur la performance collective en aviron se sont majoritairement intéressées à la coordination des mouvements des rameurs d'un point de vue mécanique et biomécanique (e.g., Baudouin \& Hawkins, 2004; Hill, 2002; Wing \& Woodburn, 1995). Les rares études en psychologie du sport relatives à la performance collective en aviron, ont quant à elles investigué des construits psychosociaux tels que la cohésion ou l'efficacité collective (e.g., Magyar, Feltz, \& Simpson, 2004; Mugford \& Tennant, 2004) sans s'attacher à la dimension cognitive de la coordination entre les rameurs.

Il semble donc que la littérature relative à l'aviron, tant professionnelle que scientifique, accorde peu d'attention aux phénomènes cognitifs de coordination interpersonnelle entre les rameurs en tant que facteurs de la performance collective. Cette dernière est essentiellement appréhendée à travers des caractéristiques individuelles relativement stables (compétences et capacités physiques, psychologiques ou techniques des rameurs), et préexistantes à l'activité collective elle-même. Cependant en sciences du sport se développe depuis quelques années un courant de recherche sur les processus dynamiques inhérents à la coordination entre les partenaires des équipes sportives (pour une synthèse voir Sève, Bourbousson, Poizat, \& Saury, 2010). Le développement de ce courant est lié au constat que ces processus ont jusqu'alors été peu étudiés par les traditions de recherche dominantes en psy- 
chologie du sport qui se sont essentiellement centrées, pour l'étude des équipes sportives, sur des phénomènes décrits par des concepts issus de la psychologie des groupes (e.g., cohésion, sentiment d'efficacité collective, degré de satisfaction), et sur des processus cognitifs individuels des sportifs (e.g., leurs stratégies perceptives et décisionnelles, leur fonctionnement mnémonique, le contenu et l'organisation de leurs bases de connaissances). Pour différents auteurs (e.g., Cooke, Stout, \& Salas, 2001; Eccles \& Johnson, 2009; Eccles \& Tenenbaum, 2004, 2007; Fiore \& Salas, 2006; Reimer, Park, \& Hinsz, 2006), ces processus de coordination interpersonnelle apparaissent pourtant cruciaux dans la compréhension de l'activité et des performances collectives: ils expliqueraient notamment qu'une « équipe experte » ne se réduise pas à une « équipe d'experts », et que la performance collective diffère de la somme des performances individuelles des membres de l'équipe. Ces auteurs ont en conséquence appelé au développement de recherches centrées sur la "coordination cognitive » au sein des équipes sportives. Ces recherches étudient le fonctionnement et les performances des équipes en accordant une place centrale aux phénomènes cognitifs permettant aux différents membres d'une équipe de se coordonner.

\section{2.- L'étude des coordinations interpersonnelles en sport}

En France, différentes études ont été conduites récemment pour appréhender ces phénomènes cognitifs. La majorité de ces études a été conduite en référence au programme scientifique et technologique du «cours d'action » (Theureau, 2006). Le cadre théorique et méthodologique d'analyse du cours d'action vise à appréhender, dans le même mouvement, les activités individuelles et l'activité collective, et à accéder à la dynamique des significations construites par les acteurs en situation. Ce cadre d'analyse propose plusieurs objets théoriques pour modéliser l'activité humaine (Theureau, 2006). L'objet théorique « cours d'expérience » est une réduction de l'activité à sa partie qui est significative pour l'acteur: le cours d'expérience est « l'activité d'un acteur engagé dans une situation, qui est significative pour ce dernier, c'est-à-dire montrable, racontable et commentable par lui à tout instant, moyennant des conditions favorables » (Theureau, 2004, p. 48). Cet objet théorique ne prétend pas rendre compte de l'ensemble des niveaux d'organisation de l'activité: il permet de décrire les phénomènes de l'activité qui sont dits «significatifs pour l'acteur » (i.e., « montrables, racontables et commentables » par lui), cette réduction étant fondée sur les postulats selon lesquels ce niveau d'organisation est suffisamment autonome par rapport aux autres pour donner lieu à des observations, descriptions et explications scientifiques valides, et que celles-ci peuvent contribuer utilement à la conception de situations.

Le cours d'expérience d'un acteur, au cours d'une période d'activité, est reconstruit sur la base de données comportementales recueillies en situation (le plus souvent des données d'enregistrement audiovisuel) et des données de verbalisation recueillies lors d'un entretien dit d'autoconfrontation (l'acteur, confronté à l'enregistrement audiovisuel, est invité à décrire et commenter ses perceptions, interprétations, les éléments pris en compte pour agir, les connaissances mobilisées). Cette méthodologie permet d'accéder à la cognition déployée par des acteurs dans des situations réelles. Le cours d'expérience est constitué d'un enchaînement d'unités d'activité significatives émergeant de l'interaction de l'acteur avec sa situation. Ces unités articulent différentes composantes dont les préoccupations de l'acteur, ses attentes, les connaissances mobilisées, les éléments pris en compte dans la situation. L'analyse de l'objet théorique «articulation des cours d'expérience » de plusieurs acteurs vise à caractériser la manière dont l'activité collective se construit sur la base de la concaténation des activités individuelles.

Les études sur la performance collective en sport menées en référence au cadre théorique du cours d'action (e.g., Bourbousson, Poizat, Saury, \& Sève, 2008; Poizat, Bourbousson, Saury, \& Sève, sous presse; Poizat, Sève, \& Saury, 2007; Poizat, Sève, Serres, \& Saury, 2008; Saury, 2008) ont appréhendé cette performance à l'aide de l'articulation des cours d'expérience des membres d'une même équipe sportive. Elles ont permis de décrire différentes formes et processus de partage cognitif permettant les ajustements respectifs entre les partenaires d'une équipe sportive et supportant la 
performance collective. À titre d'illustration, des études sur la coordination interpersonnelle en basket-ball ont analysé les réseaux de coordination cognitive entre les partenaires d'une même équipe (Bourbousson et al., 2008; Bourbousson \& Sève, sous presse). Elles ont mis en évidence que des connexions cognitives étaient plus particulièrement privilégiées entre certains membres (du point de vue de leur fréquence d'occurrence dans le cours du match), de sorte que chaque joueur s'intégrait à un réseau de coordination spécifique. L'équipe est apparue comme une entité dynamique qui se faisait et se défaisait en permanence, des sous-réseaux se constituant et disparaissant au cours du match. Des études sur les informations contextuelles partagées par des partenaires d'une équipe de double en tennis de table, ont pointé l'existence de différents types de partage (symétrique, asymétrique, non partage) et des processus visant à réguler ce partage (enquête, mise en visibilité, surveillance, masquage) (Poizat et al., 2008, sous presse). Enfin une étude sur les modalités de coopération entre régatiers au sein d'équipages de voile a mis en évidence des formes de coopération intégrant momentanément des interactions concurrentielles entre les partenaires dans la prise de décisions tactiques au cours des régates, ainsi que différents processus de régulation de la coopération (la construction d'une intelligibilité mutuelle et de jugements de confiance à l'égard du partenaire, l'influence mutuelle de l'activité et des jugements du partenaire, et la construction et l'actualisation continue d'un « statut temporaire» relatif des partenaires) (Saury, 2008).

\section{3.- Les objectifs de l'étude}

Si les études précédentes ont permis de caractériser certains des processus cognitifs participant à la coordination entre les membres d'une équipe sportive (et plus largement de toute équipe engagée dans la réalisation d'une performance collective), leur méthodologie rend cependant difficile l'accès à des processus non verbalisables susceptibles de jouer pourtant un rôle essentiel dans la coordination, notamment lorsqu'il s'agit d'activités sportives, qui exigent des ajustements sensori-moteurs fins pour la synchronisation des actions des différents partenaires. La présente étude s'inscrit dans le prolongement de ces études. Elle a été menée en collaboration avec des entraîneurs et des athlètes de haut niveau en aviron. Elle poursuivait simultanément deux objectifs : (a) améliorer la compréhension de la performance des équipages dans la perspective d'optimiser l'entraînement en aviron, et (b) esquisser de nouvelles perspectives méthodologiques visant à enrichir la compréhension des processus de coordination (au niveau significatif et non significatif de l'activité). D'un point de vue empirique, il s'agissait de décrire les phénomènes associés à la coordination entre rameurs au cours de la réalisation d'une performance en équipage, en articulant deux axes d'analyse complémentaires: une analyse des cours d'expérience des rameurs lors de courses, et une analyse de paramètres mécaniques corrélatifs de leur activité durant ces mêmes courses.

Il s'agissait, en premier lieu, de caractériser les éventuels phénomènes inhérents à l'expérience des rameurs, relatifs à leur coordination avec leur partenaire, en situation de réalisation de performance. Les rameurs prennent-ils en compte l'activité de leur partenaire au cours des épreuves d'aviron d'une façon « significative pour eux »? S'ajustent-ils à l'activité de leur partenaire? Selon quelles modalités? Partagent-ils les mêmes préoccupations, perceptions, interprétations, au cours d'une course? La réponse à ces questions devait permettre de spécifier les modalités de coordination en aviron - i.e., dans une situation sportive collective mettant en jeu une activité motrice cyclique fortement contrainte mécaniquement - et d'analyser leurs particularités éventuelles en comparaison de celles décrites par la littérature professionnelle en aviron et par la littérature scientifique relative à d'autres sports. L'activité collective a été appréhendée en termes de cours d'expérience et d'articulation des cours d'expérience.

Il s'agissait, en deuxième lieu, d'explorer la fécondité d'une analyse complémentaire à celle des cours d'expérience des rameurs, visant à prendre en considération des paramètres mécaniques rendant compte de la coordination interindividuelle entre les rameurs à un niveau non significatif (ou non verbalisable) pour eux. En effet, en aviron on peut s'attendre à ce qu'une partie importante des ajustements inter-rameurs repose sur des processus non-intentionnels et implicites (ajustements sen- 
sori-moteurs automatisés ou se manifestant à un niveau de son activité non verbalisable pour l'acteur). L'enjeu méthodologique était d'explorer en quoi le recours à certains paramètres mécaniques était susceptible d'enrichir la compréhension des modalités de coordination entre les rameurs, au niveau où elle est significative pour eux.

\section{2.- Méthode}

\section{1- Participants et situation}

Deux équipages féminins juniors de «deux de pointe sans barreur » du Pôle Espoir de Nantes ont été volontaires pour participer à cette étude, mise en œuvre de façon concertée avec leur entraîneur. Le « deux de pointe sans barreur » est une catégorie de bateaux d'aviron à deux rameurs, dans laquelle chacun dispose d'un seul aviron. Deux postes sont distingués au sein de l'équipage: le rameur situé le plus à l'arrière du bateau (par rapport au sens de déplacement) est dit « chef de nage », car c'est traditionnellement à lui que revient la responsabilité de fixer la cadence des cycles d'aviron; le rameur situé le plus à l'avant du bateau est dit « deux ». Compte tenu du placement en ligne des deux rameurs, le rameur « deux » est assis derrière le « chef de nage », qu'il a dans son champ de vision (Figure 1).

Afin de préserver l'anonymat des participantes, chaque rameuse a été désignée par un pseudonyme. L'Équipage 1 était composé de Kim (18 ans, « chef de nage ») et Maryse (17 ans, rameuse « deux »); l'Equipage 2 de Marion (18 ans, «chef de nage ») et d'Amélie 17 ans (rameuse « deux »). Ces rameuses étaient inscrites sur les listes officielles de sportifs de haut niveau. Pour chacun des deux équipages, il s'agissait de leur première saison ensemble.

L'activité des deux équipages a été étudiée lors de deux courses contre la montre sur 3000 mètres, qui sont conventionnellement désignées Course 1 (concernant l'Equipage 1) et Course 2 (concernant l'Equipage 2) dans la suite de l'article. Ces courses étaient organisées dans le cadre ordinaire de la préparation sportive des deux équipages: il s'agissait dans les deux cas d'un test précompétitif chronométré, réalisé quatre jours avant une épreuve contre la montre sur un parcours de 6000 mètres, représentant un enjeu très important dans la saison sportive des deux équipages.

\section{2.- Recueil des données}

Deux dispositifs de recueil des données ont été mis en œuvre, visant respectivement, à (a) documenter les cours d'expérience des rameuses, et (b) calculer un ensemble de paramètres mécaniques relatifs à la coordination entre les rameuses.

\subsection{1.- Recueil des données pour l'analyse des cours d'expérience}

Les comportements et communications des rameuses (équipées de micros-émetteurs HF) ont été enregistrés pendant l'intégralité des courses (d'une durée d'environ 12 minutes), grâce à deux caméras respectivement connectées à un récepteur HF. La course a été filmée par deux des coauteurs à partir d'un bateau suiveur, selon un plan de cadrage en trois-quarts arrière, incluant au premier plan l'ensemble du bateau et les deux rameuses, et en arrière-plan, le défilement de la berge.

Des entretiens d'autoconfrontation ont été organisés immédiatement après les deux courses, dans deux salles du Pôle d'entraînement, selon les principes généraux de cette méthode dans le cadre du programme du cours d'action (Theureau, 2006). Chaque rameuse a été individuellement confrontée à l'enregistrement audiovisuel de la course, et incitée à se « remettre dans sa course » afin de décrire et commenter chronologiquement et de façon détaillée son expérience au cours de son déroulement. Deux chercheurs ont conduit séparément mais simultanément les entretiens. Chacun aidait la rameuse à préciser l'explicitation et la description de son expérience à chaque instant de la course grâce à des relances portant sur ses sensations (e.g., comment te sens-tu à ce moment?), ses perceptions (e.g., qu'est-ce que tu perçois?), ses focalisations (e.g., à quoi fais-tu attention ?), ses préoccupations 
(e.g., qu'est-ce que tu cherches à faire ?), ses émotions (e.g., qu'est-ce que tu ressens ?), et ses pensées et interprétations (e.g., qu'est-ce que tu penses ?). Le chercheur et la rameuse pouvaient interrompre le déroulement du visionnement de l'enregistrement, ou opérer des retours en arrière, grâce à une télécommande disposée entre les deux protagonistes, afin de faciliter la description des phénomènes de l'activité et des événements significatifs pour la rameuse,

Les entretiens qui ont suivi la Course 1 ont respectivement duré 55 minutes (Kim) et 49 minutes (Maryse); et ceux qui ont suivi la Course 2, respectivement 1 heure six minutes (Marion) et 1 heure dix minutes (Amélie). Chaque entretien a été intégralement enregistré à l'aide d'une caméra numérique, cadrée sur l'écran du micro-ordinateur servant au visionnement, afin de faciliter une mise en relation ultérieure précise des événements de la course et des commentaires en autoconfrontation.

\subsection{2.- Mesures mécaniques}

Les données mécaniques ont été recueillies lors de la Course 2 avec l'Equipage 2 (Marion-Amélie), grâce au système Powerline (Peach Innovations), spécialement conçu pour réaliser des mesures embarquées en aviron. Ce système comprenait une centrale d'acquisition et de stockage de données reliée à différents capteurs (Figure 1): deux capteurs de force mesurant les forces exercées sur les dames de nage (accessoires supportant l'aviron, articulés autour d'un axe vertical, permettant la rotation des avirons) dans l'axe d'avancement du bateau, deux capteurs goniométriques mesurant l'angle de balayage horizontal des avirons, un accéléromètre placé dans l'axe longitudinal du bateau, et un capteur de vitesse du bateau (capteur à hélice fixé sous le bateau). Léger, peu encombrant, facile et rapide à installer et désinstaller dans les conditions ordinaires de l'entraînement, ce système était déjà utilisé en routine à l'entraînement que ce soit lors des stages des équipes de France olympique (Barré \& Gossé, 2007) ou au sein des Pôles France et Espoir de Nantes. Les angles de chaque aviron dans le plan horizontal, les forces appliquées sur les dames de nages dans l'axe de déplacement du bateau, la vitesse et l'accélération du bateau, ont été mesurés avec une fréquence d'acquisition de 50 $\mathrm{Hz}$ tout au long de la course.

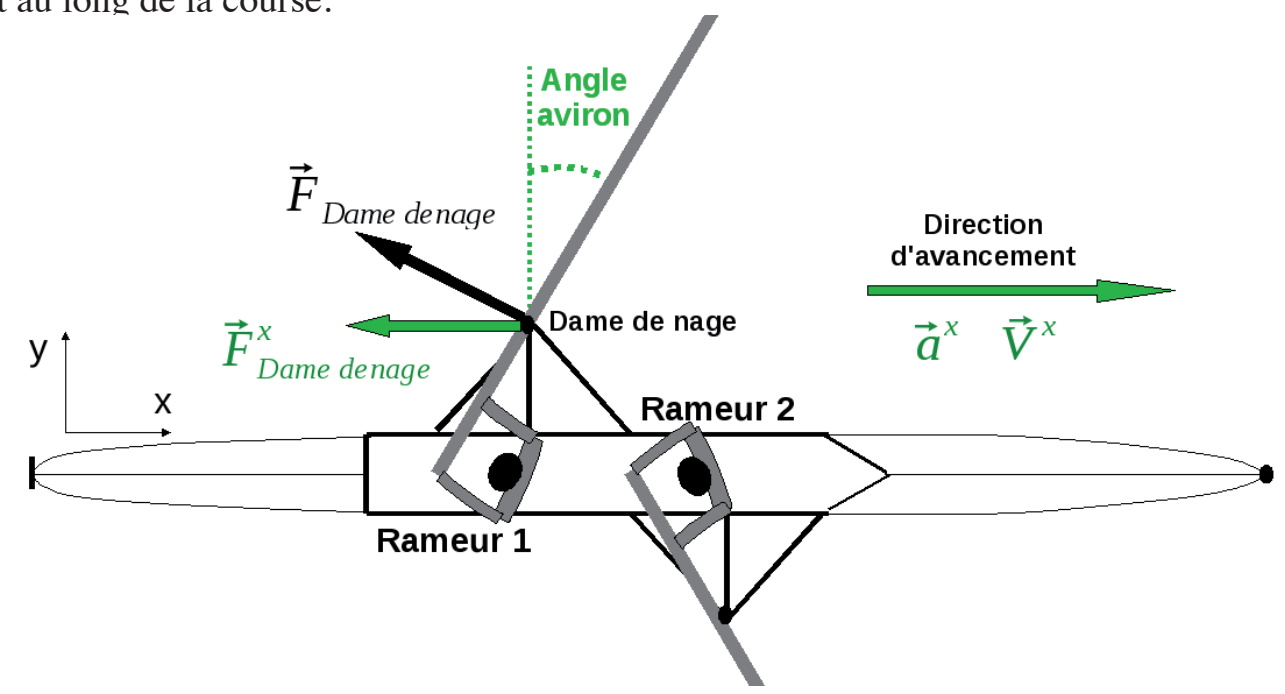

Figure 1: Vue du dessus d'un « deux de pointe sans barreur ». Le système de mesure embarqué utilisé (Powerline, Peach Innovation) permet, pour chaque rameur, de mesurer les composantes suivant l'axe x (dans la direction d'avancement du bateau) de la force appliquée par le rameur sur la dame de nage, de l'accélération et de la vitesse du bateau, ainsi que l'angle formé par l'aviron par rapport à l'axe y (perpendiculaire à l'avancement du bateau).

Figure 1: Bird's-eye view of a coxless pair. The onboard measurement system (Powerline, Peach Innovation) records the components of the forces applied by the rowers to the oarlock along the $x$-axis (in the direction of the boat's movement), the acceleration and speed of the boat, and the angle formed by the oar with the y-axis (perpendicular to the boat's movement). 


\section{3.- Analyse des données}

L'analyse des données a été conduite selon trois grandes étapes, visant respectivement, à (a) caractériser les cours d'expérience des rameuses et l'articulation collective de ces cours d'expérience; (b) calculer des paramètres mécaniques rendant compte de la coordination entre les rameuses; et (c) croiser l'analyse des cours d'expérience et des paramètres mécaniques pour certains moments critiques dans le déroulement de la course, du point de vue de la coordination entre les rameuses.

\subsection{1.- Analyse des cours d'expérience}

Dans un premier temps, les données d'enregistrement in situ et en autoconfrontation ont été intégralement transcrites et ont donné lieu à la construction de chroniques d'activité pour chaque équipage. Celles-ci ont été organisées sous la forme d'un tableau à quatre colonnes: la première colonne restituait le déroulement du temps de la course; la deuxième colonne les communications verbales et les mouvements de la tête des rameuses (retournements pour contrôler la direction) durant la course ; la troisième et la quatrième colonne les extraits des verbalisations en autoconfrontation correspondant à ces moments, respectivement pour chacune des deux rameuses de l'équipage (Tableau 1).

\begin{tabular}{|c|c|c|c|}
\hline Temps & $\begin{array}{l}\text { Communications } \\
\text { et comportements }\end{array}$ & Autoconfrontation Kim & Autoconfrontation Maryse \\
\hline 0’00» & Départ & $\begin{array}{l}\text { Chercheur: Donc départ lancé... } \\
\text { les premiers coups de rame... } \\
\text { Kim: C'est parti ensemble, ça } \\
\text { allait, je me suis dit c'est parti! } \\
\text { Chercheur: Des sensations parti- } \\
\text { culières...? } \\
\text { Kim : Moins lourd que d'habi- } \\
\text { tude... } \\
\text { Chercheur: Là sur les deux } \\
\text { premiers coups de rames... } \\
\text { Kim: C'était bien ensemble, } \\
\text { c'était bien... }\end{array}$ & $\begin{array}{l}\text { Chercheur: Alors là (moment du départ) } \\
\text { Maryse : Là c'est vrai qu'on a eu du mal à se } \\
\text { trouver, puisqu'on a eu... enfin je ne savais pas } \\
\text { que Kim allait partir comme ça [aussi rapide en } \\
\text { cadence] je pensais qu'elle allait être plus basse } \\
\text { en cadence... alors que moi j'étais en avance } \\
\text { sur elle, et là je me suis étonnée à être un petit } \\
\text { peu en retard... au départ, ouais... je pensais pas } \\
\text { qu'elle allait partir aussi vite... } \\
\text { [...] j'étais surprise au départ... j'étais partie } \\
\text { moins vite, enfin dans ma tête, je partais pour } \\
\text { être moins rapide en cadence... et donc heu, sur } \\
\text { les premiers coups je me suis laissée un peu } \\
\text { emporter, enfin c'est elle qui m'a un peu... ouais } \\
\text { je me sentais en retard, en fait... }\end{array}$ \\
\hline 0'15» & $\begin{array}{l}\text { (Kim tourne la tête à } \\
\text { gauche) } \\
\text { Kim: «Longueur!» }\end{array}$ & $\begin{array}{l}\text { Chercheur: Et là quand tu dis } \\
\text { « longueur » } \\
\text { Kim: Parce qu'on était un peu } \\
\text { trop en trois quart on n'était pas } \\
\text { encore en longueur... là on était } \\
\text { encore en trois quart... C'est pour } \\
\text { qu'on se cale ensemble! }\end{array}$ & $\begin{array}{l}\text { Chercheur: d'accord... et là, bon, c'est parti } \\
\text { mais qu'est-ce que tu cherchais à faire? } \\
\text { Maryse : Là c'est plus du technique, donc là [le } \\
\text { but], c'était bien replacer... plus rapidement parce } \\
\text { que moi j'avais tendance à m'arrêter un petit } \\
\text { peu, pour essayer justement d'être bien ensemble } \\
\text { avec elle avant, sur les séries d'entraînement } \\
\text { [...] pour essayer justement d'être bien ensemble } \\
\text { avec elle... [...] ben là justement, j'essaye de } \\
\text { replacer plus vite pour, justement réussir à garder } \\
\text { son rythme, à Kim. }\end{array}$ \\
\hline
\end{tabular}

Tableau 1 : Extrait de chronique d'activité de l'Equipage 1 (Kim et Maryse) au départ de la Course 1.

\section{Table 1: Extract from the activity log of crew 1 (Kim and Maryse) at the start of Race 1.}

Dans un deuxième temps, les cours d'expérience des deux rameuses ont été documentés: les phénomènes significatifs pour elles liés à leur coordination (en termes de préoccupations, d'attentes, de connaissances mobilisées, de perceptions et d'interprétations) ont été repérés en référence aux catégories génériques analytiques du signe hexadique (Theureau, 2006). Le signe hexadique est consti- 
tué de six composantes, rendant respectivement compte des phénomènes suivants de l'expérience humaine: (a) les préoccupations de l'acteur à l'instant t: l'engagement (noté E); (b) les attentes de l'acteur dans la situation à l'instant t compte tenu de son engagement: l'actualité potentielle (notée A); (c) les connaissances mobilisées par l'acteur compte tenu de son engagement et de ses attentes à l'instant t: le référentiel (noté S); (d) les jugements perceptifs ou proprioceptifs de l'acteur à l'instant t (i.e., les éléments de la situation qui «font signe » pour lui dans la situation): le représentamen (noté R); (e) la fraction de son activité significative pour l'acteur à l'instant t (action, communication, focalisation, interprétation ou sentiment) : l'unité de cours d'expérience (notée U); et (f) le processus de validation ou d'invalidation de connaissances antérieures, ou de construction de nouvelles connaissances à l'instant $\mathrm{t}$ : l'interprétant (noté I) (Tableau 2).

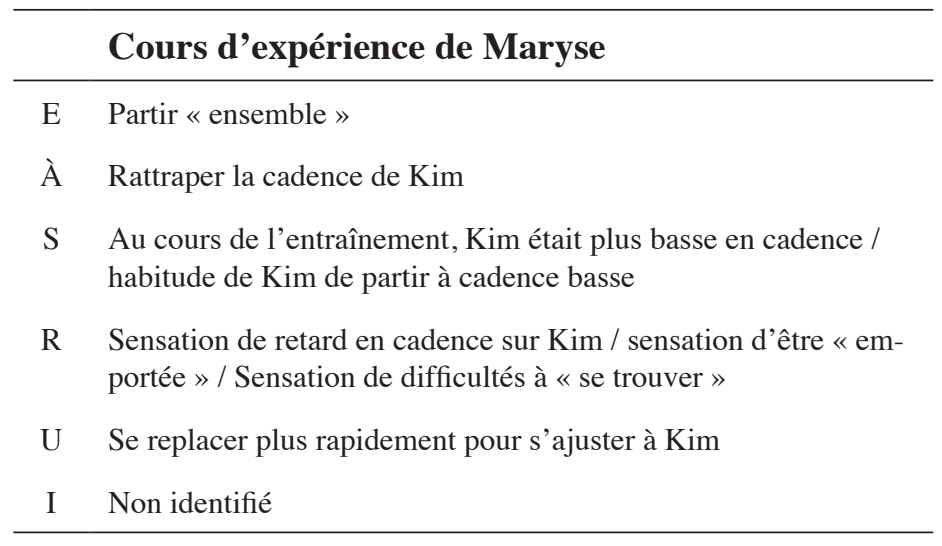

Tableau 2: Extrait du cours d'expérience Kim au départ de la Course 1.

Table 2: Extract from Kim's course of experience at the start of Race 1.

Dans un troisième temps, les cours d'expérience des deux rameuses ont été mis en correspondance temporelle, permettant de comparer à chaque instant du déroulement de la course, la similitude ou la congruence de leurs préoccupations, attentes, perceptions et interprétations.

Dans un quatrième temps nous avons identifié les phénomènes significatifs pour les rameuses liés à leur coordination. Cette identification a été réalisée sur la base d'une analyse compréhensive des cours d'expérience des rameuses et de leur articulation. Cette analyse a donné lieu à l'identification de trois phénomènes significatifs relatifs à la coordination entre les rameuses.

\subsection{2.- Paramètres mécaniques calculés}

Les mesures mécaniques réalisées permettaient a priori de calculer un grand nombre de paramètres mécaniques différents. Parmi ceux-ci, certains ont été sélectionnés sur la base d'un examen des études de mécanique et de biomécanique relatives à la performance en équipage d'aviron (Baudouin \& Hawkins, 2004; Hill, 2002; Hill \& Fahrig, 2009; Hofmijster, Landman, Smith, \& Van Soest, 2007), et en concertation avec les entraîneurs. Ces paramètres ont été calculés par coup d'aviron (les rameuses réalisaient environ 350 coups pour une course d'une durée de 12'30", correspondant à une cadence moyenne de 28 coups par minutes). Ils ont été choisis afin de rendre compte de trois sortes de phénomènes potentiellement significatifs pour les rameuses: les performances individuelles de chaque rameuse (e.g., durée et vitesse des coups d'aviron, puissance produite), la performance globale de l'équipage (e.g., variations de vitesse du bateau), et l'état de la synchronisation des mouvements des deux rameuses (e.g., différences d'amplitude et décalages temporels entre les mouvements d'avirons).

\subsection{3.- Croisement des analyses des cours d'expérience et des analyses mécaniques}

Le croisement des analyses des cours d'expérience et des paramètres mécaniques a été réalisé en 
respectant le principe d'un «primat du cours d'expérience » (Theureau, 2006), c'est-à-dire dans le cadre d'une démarche subordonnant l'analyse mécanique à la compréhension des phénomènes mis à jour par l'analyse de l'articulation des cours d'expérience. L'analyse de l'articulation des cours d'expérience a permis de mettre en évidence des moments particulièrement « saillants » ou « critiques » survenant durant la course, du point de vue du vécu des rameuses, concernant la performance collective (sensations d'efficacité, de glisse... vs moindre efficacité, bateau lourd...), ou la coordination inter-rameuses (e.g., sensations d' « être ensemble » ou de « ne pas être ensemble », sensations d'avance ou de retard sur sa partenaire au cours du mouvement de rame). Ces moments particuliers de l'activité collective ont alors fait l'objet d'une enquête ciblée visant à rendre compte de leurs corrélats mécaniques.

\section{3.- Résultats}

Les résultats sont présentés dans deux sections. La première thématise les phénomènes significatifs pour les rameuses liés à leur coordination, issus de l'analyse des cours d'expérience et de leur articulation. La deuxième présente une étude de cas mettant en jeu le croisement des analyses du cours d'expérience et de paramètres mécaniques rendant compte de caractéristiques de la coordination inter-rameuses, afin de révéler la fécondité d'une telle démarche pour comprendre cette coordination.

\section{1.- Les phénomènes significatifs pour les rameuses liés à leur coordination}

Trois phénomènes significatifs pour les rameuses liés à leur coordination ressortent de l'analyse: (a) une sensibilité particulière à l'état de la coordination, (b) une interprétation récurrente de l'activité de sa partenaire, et (c) quatre modalités typiques d'ajustements mutuels.

\subsection{1.- Une sensibilité particulière à l'état de la coordination : « être ensemble... »}

Parmi les phénomènes significatifs de leur activité durant la course, ceux qui ont fait l'objet du plus grand nombre de commentaires de la part des rameuses au cours des entretiens d'autoconfrontation concernaient leur préoccupation constante de rester «bien ensemble» tout au long de la course, et leurs perceptions « d'être ensemble » ou de « ne pas être ensemble » selon les moments de son déroulement. Pour les quatre rameuses, la performance de l'équipage était étroitement subordonnée au maintien de cet état de la coordination, perpétuellement menacé durant la course, et qui faisait l'objet d'incitations verbales fréquentes par la rameuse « chef de nage » à l'attention de sa coéquipière ( « ensemble! ») au cours même du déroulement de la course. Cet état était décrit d'une façon syncrétique par les rameuses e.g., Course 2, Amélie (au départ): « je sais pas comment expliquer enfin... on était, on était ensemble... le bateau il glissait bien... »; Marion (aux mille mètres): "à partir de là, $j$ 'ai senti qu'on n'était plus ensemble Amélie et moi... paf on tombe dans l'eau et après on envoie... Je sentais que là soit elle était en avance sur moi soit en retard. »). Cependant, il était associé à des indices perceptifs et proprioceptifs variés, respectivement, (a) sur soi, (b) sur sa partenaire, (c) sur le bateau, et (d) sur la synchronisation du mouvement des avirons.

Les indices sur soi concernaient les sensations corporelles éprouvées par les rameuses au cours de la réalisation de leurs mouvements de rame. Par exemple, lors de la Course 2, Marion a exprimé la perception récurrente d'être "poussée sur l'avant » par Amélie dans la réalisation de son mouvement de rame, ce qu'elle associait au fait de «ne pas être ensemble »: "j'ai vu aussi qu'on n'était pas ensemble parce que [...] je me sentais en fait poussée...». Puis, quelques instants plus tard, percevant une coordination plus efficace, elle a précisé: "là c'est... je l'ai plutôt senti au niveau du corps... enfin, je me sentais moins poussée. [...] on repartait ensemble...».

Les indices sur sa partenaire concernaient les éléments significatifs pour chaque rameuse liés aux comportements de sa coéquipière, révélant un état de la coordination. Par exemple, lors de la Course 1, Kim a perçu que Maryse avait des difficultés pour respirer alors que cette dernière était « en 
avance » sur elle et qu'elle avait tendance à la « pousser ». A ce moment de la course, Kim a incité Maryse à respirer plus amplement afin d'augmenter l'amplitude de son mouvement d'aviron (elle lui a crié pendant la course "ensemble! On respire Maryse allez! »), ce qu'elle a commenté dans les termes suivants en autoconfrontation: "Elle était en avance [...] Je sentais qu'elle était... qu'elle respirait plus trop bien là... [...] là j'ai entendu qu'elle avait du mal à respirer...»).

Les indices sur le bateau concernaient les répercussions, perçues par les rameuses du fait d' «être ensemble » ou de «ne pas être ensemble », sur la glisse de la coque, ou sur son équilibre latéral ou longitudinal. Par exemple, lors de la Course 2, Marion a associé le fait de «ne pas être ensemble » dès le départ de la course à des mouvements de la coque nuisibles à une bonne glisse du bateau: "là dès le premier coup je me dis ben là ça va pas... [...] on n'est pas sorties ensemble et donc le bateau tout de suite il commence à bouger et on l'a pas propulsé ensemble, c'est ça le problème [...] Le bateau il bouge dans tous les sens...». D'une manière générale, l'état «être ensemble » était associé par les rameuses à une sensation de fluidité et de glisse sur l'eau du bateau.

Les indices sur la synchronisation du mouvement des avirons concernaient les perceptions par les rameuses du caractère synchrone ou asynchrone des mouvements à des moments caractéristiques du cycle de rame: à la «tombée » des pelles dans l'eau (moment de la «prise d'eau » au début de la phase propulsive), et/ou lors du retour aérien des rames (phase non propulsive, durant laquelle les rameuses se replacent pour prendre un nouvel appui dans l'eau). Par exemple, dans la Course 2, Marion a perçu dès les premiers coups d'aviron après le départ qu'Amélie effectuait son attaque très légèrement avant elle: "Elle tombait avant moi dans l'eau, sa rame, sa pelle tombait avant moi dans l'eau... », ce qu'elle associait à la sensation corporelle concomitante "d'être poussée » dans son mouvement de rame. Ce décalage temporel, qui engendrait un sentiment d'urgence chez la rameuse dans la réalisation de son mouvement de rame, était mis en relation avec sa perception concomitante de pas pouvoir exploiter toute l'amplitude de ce mouvement, comme elle l'a exprimé lors de l'autoconfrontation (Marion: "j'avais l'impression de pas être en longueur du tout parce qu'en fait on a un petit repère, on a une paille et on doit arriver à cette paille-là avant de mettre la pelle dans l'eau [...] et le problème c'est que moi j'arrivais pas à l'atteindre et alors qu'Amélie était avant moi... elle partait dans l'eau avant moi »).

Ces indices, pris en compte de façon concomitante dans la plupart des cas, permettaient ainsi aux rameuses d'actualiser constamment leur jugement d' «être ensemble» ou de «ne pas être ensemble» à chaque instant de la course. Ce jugement évoluait tout au long de la course et pouvait être ou non partagé par les deux rameuses. À titre d'illustration lors du début de la Course 1, Kim avait la sensation « d'être ensemble » alors que Maryse avait la sensation d'être en retard par rapport à Kim. Au bout de 100 mètres de course, les deux rameuses partageaient le jugement « être ensemble ».

\subsection{2.- Une interprétation récurrente de l'activité de sa partenaire}

Au cours des deux courses, les quatre rameuses ont fait preuve d'une surveillance récurrente de l'activité de leur partenaire et construit des interprétations relatives à cette activité. Celles-ci leur permettaient d'inférer les préoccupations, l'état de fatigue, l'état émotionnel ou l'intensité de l'investissement de leur coéquipière, au fur et à mesure des événements de la course, en relation avec la connaissance de cette coéquipière issue d'expériences communes passées.

Par exemple, dès le début de la Course 1, Maryse (rameuse « deux ») a reconnu, dans la fréquence avec laquelle sa coéquipière (Kim, rameuse « chef de nage ») se retournait, une préoccupation teintée d'inquiétude de cette dernière concernant le contrôle de la direction du bateau ${ }^{1}$. Lors de l'autoconfrontation, Maryse a indiqué que quand Kim tournait la tête aussi fréquemment (ce qui pénalise $a$ priori l'efficacité du mouvement de rame), cela indiquait « soit qu'elle arrive plus à se concentrer, soit c'est qu'elle est perdue dans l'espace... parce que normalement on peut la voir [la direction] au niveau de la pointe avant [...] ça c'est, soit qu'elle se perd, elle veut voir ce qui se passe derrière,

1. Compte-tenu des positions respectives des rameuses dans le bateau, c'est à la rameuse « deux » qu'incombe ordinairement le rôle de se retourner afin de surveiller les écarts de direction du bateau. 
donc où... où sont les bouées, par exemple [...] et moi là c'est... je perçois ça comme... pff... soit un signe en me disant que [...] ouais, ben là c'est parce qu'elle était perdue... ».

Au cours de cette même course, Kim a évoqué l'état de fatigue de Maryse, qu'elle a inféré à la micourse (environ 1500 mètres), sur la base de la perception d'un déséquilibre directionnel du bateau: " [je dis] 'léger devant!' ...ça commençait à se durcir... et puis à ce moment-là je 'tournais ${ }^{2}$ 'Maryse en fait... j'avais beau avoir la barre qui tournait on allait toujours dans cette direction (montre la berge) [...] Je tirais trop fort, enfin moi je tirais mais... elle devait être fatiguée... je le sens comme c'est moi qui ai la direction ... elle se durcissait du coup elle était moins rentable...».

Durant la Course 2, Marion a évoqué la « peur des vagues » qu'elle percevait chez Amélie avant même le départ de la course, l'incitant à la rassurer au moment où elles s'apprêtaient à prendre le départ (Marion: "fais-moi confiance »), moment qu'elle a ensuite commenté dans les termes suivants en autoconfrontation: "[je lui dis] 'fais-moi confiance' parce que je la sens trembler dans le bateau... Mais ça à tous les entraînements je la sens trembler [...] je sens le bateau qui bouge un tout petit peu, et je sais que c'est elle parce qu'après quand je me retourne un peu, je vois que c'est elle, elle est pas rassurée alors elle tremble... et elle s'en rend pas compte en fait [...] je pense qu'elle a peur d'aller à l'eau et donc c'est pour ca, donc je lui dis 'fait moi confiance, je suis là' [...] Amélie elle a très peur de ça [...] un 'deux sans barreur' c'est un bateau qui n'est pas stable du tout donc elle n'est pas rassurée ».

Au cours de cette même course, Marion a interprété certains moments au cours desquels elle percevait qu'elle et Amélie n'étaient plus « ensemble » en évoquant sa perception d'un degré d'attention et d'application insuffisants d'Amélie dans le contrôle de ses mouvements de rame, cette dernière ne lui semblant "plus dans le bateau » selon ses propres termes, et manifestant un investissement moins intense dans la course: "c'est mou derrière... c'est pas... il y a pas cette envie d'aller chercher loin d'aller... d'être des guerrières si on peut dire [...]...».

\subsection{3.- Quatre modalités typiques d'ajustements mutuels}

Les rameuses ont manifesté des préoccupations visant à ajuster mutuellement leurs mouvements d'aviron selon des modalités diversifiées tout au long de l'épreuve, en relation avec leurs perceptions respectives du fait «d'être ensemble » ou « de ne pas être ensemble », et de leurs interprétations de l'activité de leur coéquipière. L'analyse a mis en évidence quatre modalités typiques d'ajustements mutuels, respectivement caractérisées par les préoccupations suivantes: (a) «Etre une référence stable pour sa coéquipière »; (b) «Inciter sa coéquipière à s'ajuster à soi »; (c) « S'ajuster à sa coéquipière »; et (d) « Ramer ensemble au service du bateau ».

La préoccupation «Etre une référence stable pour sa coéquipière » consistait pour les rameuses à se focaliser intensément et quasi-exclusivement sur leur propre mouvement de rame, et à rechercher la plus grande « justesse technique » possible. De leur point de vue, le fait de bien pouvoir «poser son geste », de se concentrer sur tous les « points techniques » essentiels du mouvement d'aviron et de produire un cycle invariable, devait permettre à leur coéquipière de se synchroniser plus facilement avec elle. Cette préoccupation est apparue par exemple dans l'activité de Marion (rameuse « chef de nage »), durant la Course 2. Après avoir tenté de « lancer une série » (i.e., provoquer une accélération momentanée de la cadence de rame de l'équipage), et perçu qu'Amélie ne «suivait pas » l'accélération qu'elle souhaitait impulser et qu'elles n'étaient plus « ensemble », elle s'est alors focalisée sur son propre mouvement de rame, commentant ainsi son activité à ce moment: " j'essaie de bien... me centrer sur le replacement puisque je savais qu'Amélie ça lui fait du bien et puis ça fait du bien au bateau, enfin ça fait avancer le bateau comme ça... [...] de bien replacer, de bien enrouler sur l'arrière et de rythmer [...] bien les jambes plaquées et enrouler les mains vite... de replacer le dos sur les jambes tendues [...] décomposer mais de façon rapide on peut dire... dynamique [...] un rythme régulier, c'était ca surtout moi mon [...] [j'étais concentrée] sur moi d'abord parce que... après je

2. Dans le vocabulaire des rameurs, cette formule signifie que cette rameuse estimait exercer une puissance plus forte que sa coéquipière à ce moment, au point de créer un déséquilibre entre les deux bordées faisant tourner le bateau. 
me suis dis comme Amélie me suit bon... elle va me suivre, enfin si moi je le fais bien, elle va le faire bien!».

La préoccupation «Inciter sa coéquipière à s'ajuster à soi » consistait à chercher à influencer le comportement de sa coéquipière, au moyen d'injonctions verbales incitatives adressées à cette dernière au cours même de la course (e.g., «longueur! », « on plaque les jambes! », « on enroule! ", «laisse glisser!»). Par exemple, dans le deuxième mille mètres de la Course 1, Kim a crié à Maryse «te couche pas derrière! », avant de commenter ainsi cette intervention au cours de l'autoconfrontation: "'te couche pas derrière...' Quand elle se couche [inclinaison excessive du tronc en arrière à la fin du mouvement de propulsion] ça s'arrête derrière... et puis je sais que quand elle ne se couche pas derrière, ça fait du bien au bateau [...] là il y avait moins d'enchaînement derrière et le bateau s'enfonçait...».

La préoccupation «S'ajuster à sa coéquipière » consistait pour les rameuses à chercher à répondre, soit à une incitation verbale de leur coéquipière, soit à la perception d'une demande implicite de celle-ci. Par exemple, au cours du premier 1000 mètres de la Course 2, Marion a crié « longueur! », incitant Amélie à modifier son mouvement de rame, ainsi qu'elle l'a décrit lors de l'autoconfrontation: " là elle dit 'longueur' parce que j'étais pas assez. 'longue' [...] j'arrivais pas à aller sur l'avant [...] là, à peu prés depuis deux ou trois coups avant là... [...] [donc je cherche à] aller plus... sur l'avant, enfin bien replacer et... la suivre bien et aller plus sur l'avant, parce qu'en fait elle avait l'impression à chaque fois que je tombais avant elle... ». L'exemple suivant, choisi au cours de la Course 1, décrit un ajustement de Maryse à une demande implicite de Kim, liée à la cadence choisie par cette dernière (occupant la position de rameuse «chef de nage ») au moment du lancement du bateau au départ: "là à ce moment-là... je commence à sentir le rythme, je vois ce qu'elle veut faire, et donc là le but c'est de... c'est de suivre [...] mais j'étais surprise au départ...j'étais partie moins vite, enfin dans ma tête, je partais pour être moins rapide en cadence... et donc heu, sur les premiers coups je me suis laissée un peu emporter [...] je me sentais en retard [...] donc là [le but], c'était bien replacer... plus rapidement [...] pour essayer justement d'être bien ensemble avec elle...».

La préoccupation « Ramer ensemble au service du bateau » consistait pour les rameuses à ajuster leurs mouvements d'aviron en étant essentiellement focalisées sur les comportements et les performances du bateau. Les rameuses cherchaient à restaurer ou à préserver l'état dynamique global du fonctionnement du bateau, se concrétisant par une qualité de glisse, et des variations de direction ou d'équilibre. Cette préoccupation s'exprimait en particulier par des incitations des rameuses adressées non plus seulement à leur coéquipière, mais à l'équipage considéré comme un tout. Par exemple, dans le premier mille mètres de la Course 1, Maryse a crié « on laisse glisser ! », intervention qu'elle a commenté dans les termes suivants en autoconfrontation: " là je dis 'on laisse glisser'... et ça je m'en souviens parce que je sentais le bateau qui faisait des bonds... qui s'arrêtait à chaque fois [...] donc ça il fallait qu'on calme un peu la coulisse, qu'on arrête de mettre autant de force, qu'on laisse bien glisser, et donc moi je lui ai dit [...] [il faut être] plus légères et en même temps, ben laisser bien glisser la coque, enfin, l'énergie qu'on met il faut bien l'utiliser... ».

\section{2.- Croisement des analyses du cours d'expérience et de paramètres mécaniques selon un "primat du cours d'expérience »: une étude de cas}

Cette étude de cas concerne un moment de la Course 2 vécu par Marion (rameuse « chef de nage») comme un dysfonctionnement de la coordination de l'équipage. Du fait de la répartition prédéfinie des rôles dans le bateau, Marion avait en charge d'imprimer le rythme à l'équipage. Lors de l'entretien d'autoconfrontation, celle-ci a exprimé sa perception récurrente d' être poussée » par sa coéquipière, Amélie, et de ne pas pouvoir réaliser pleinement son mouvement, sous la pression physique de sa partenaire. L'analyse des cours d'expérience des deux rameuses a montré que cette perception n'était pas partagée entre Marion et Amélie. Ce phénomène a donné lieu au test de trois hypothèses relatives aux ajustements comportementaux des rameuses pouvant expliquer ces perceptions, et susceptibles d'être confirmées par les mesures mécaniques: 
- la première hypothèse concernait un décalage temporel entre les deux rameuses à l'attaque du mouvement d'aviron (début de la phase de propulsion, Figure 2-A), la perception « d'être poussée » de Marion pouvant être liée à une avance de sa coéquipière sur elle à ce moment (i.e., Amélie entrait son aviron dans l'eau et amorçait la phase de propulsion avant Marion);

- la deuxième hypothèse concernait une différence d'amplitude du mouvement entre les deux rameuses (qu'il s'agisse de l'amplitude globale du mouvement de balayage de l'aviron ou plus spécifiquement de l'amplitude à l'attaque, Figure 3-A). La perception de Marion pouvait s'expliquer par une amplitude globale moins grande du mouvement d'aviron d'Amélie, ou par une amplitude moins grande d'Amélie à l'attaque donnant à Marion la sensation de ne pas pouvoir réaliser son geste dans une amplitude maximale;

- la troisième hypothèse concernait une différence de vitesse sur le retour aérien du mouvement d'aviron entre les deux rameuses, la perception de Marion pouvant être liée à un mouvement de replacement plus rapide d'Amélie sur la coulisse, « pressant » ainsi le replacement de Marion, placée juste devant elle.

Ces trois hypothèses ont été jugées comme étant les plus crédibles pour expliquer cette perception de Marion, et cela a été confirmé par les entraîneurs du pôle France-Espoir de Nantes. Les évolutions temporelles des paramètres mécaniques qui ont permis de tester ces hypothèses sont présentées par la suite.

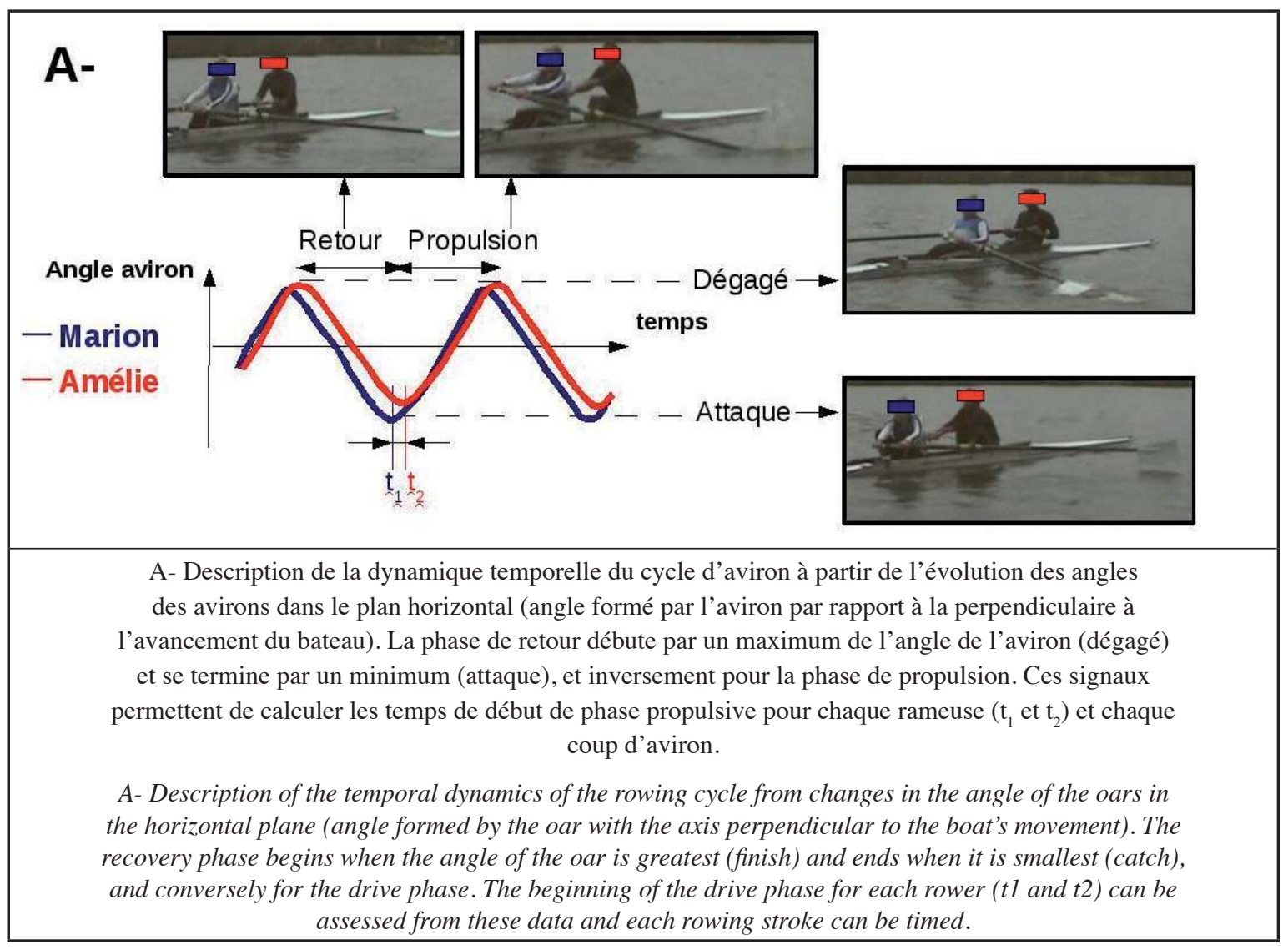




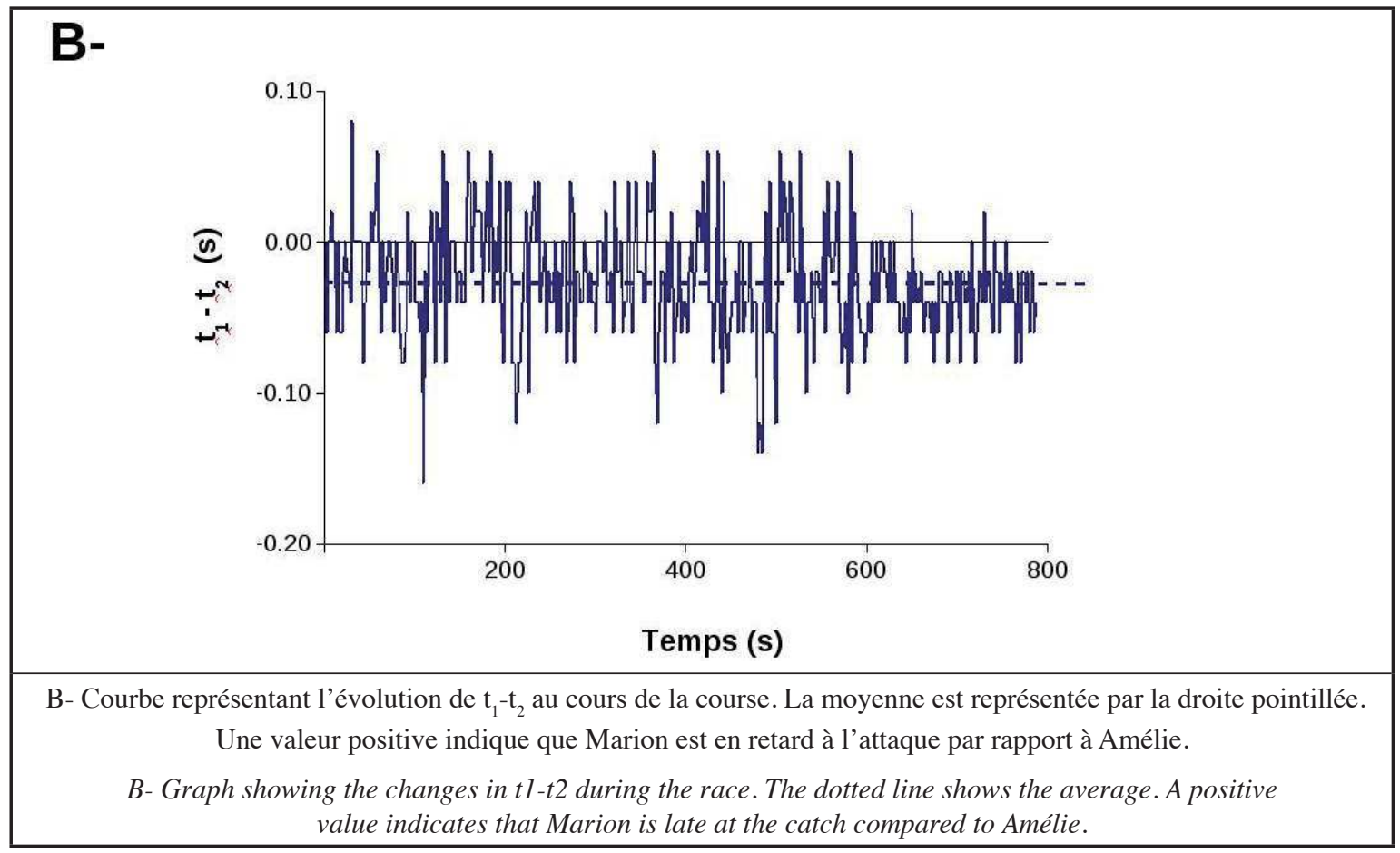

Figure 2: Test de la première hypothèse.

Figure 2: Testing the first hypothesis.

L'analyse des différences temporelles à l'attaque (début de la phase de propulsion, Figure 2), mesurées pour chaque coup d'aviron tout au long de la course, et moyennées sur la totalité de la course (Figure 2-B), a montré que Marion était plutôt en avance à l'attaque par rapport à Amélie (moyenne \pm écart type de $\mathrm{t} 1-\mathrm{t} 2$ : $-0.026 \pm 0.038$, où $\mathrm{t} 1$ et $\mathrm{t} 2$ sont les temps d'inversion des rameuses « un » [Marion] et «deux » [Amélie] respectivement). Ce résultat invalide la première hypothèse, car la perception de Marion «d'être poussée » par Amélie aurait logiquement dû être associée à une avance de cette dernière à l'attaque, ce qui n'a pas été corroboré par l'analyse mécanique. Soulignons que ce résultat est en désaccord avec l'interprétation proposée par Marion lors de l'autoconfrontation de sa perception d'être poussée par Amélie ("j'avais l'impression de pas être en longueur du tout [...] Amélie était avant moi... elle partait dans l'eau avant moi »).

L'analyse des différences d'amplitudes globales des mouvements de balayage des avirons des deux rameuses (Figure 3) a mis en évidence qu'Amélie avait un mouvement globalement moins ample que celui de Marion de $3.7 \pm 2.6^{\circ}$ (Figure 3-B). Par ailleurs, l'analyse plus ciblée de l'amplitude à l'attaque (Figure 3-C) a montré que cette amplitude était de $5.1 \pm 2.7^{\circ}$ plus importante pour Marion que pour Amélie, et que la variabilité des mesures d'amplitude à l'attaque était sensiblement plus grande pour Amélie que pour Marion. Ces résultats accréditent la deuxième hypothèse: les différences d'amplitude mesurées, notamment à l'attaque, pourraient expliquer en partie la sensation de Marion «d'être poussée » par sa coéquipière. Cette sensation était probablement amplifiée par l'importante variabilité de l'amplitude à l'attaque d'Amélie (Figure 3-C). La Figure 3 montre également que l'amplitude à l'attaque d'Amélie tendait par périodes vers des valeurs proches de celles de Marion. La transcription des communications verbales des rameuses lors de la course a montré que ces périodes coïncidaient avec des instants où Marion demandait explicitement à Amélie d'augmenter son amplitude de mouvement. 


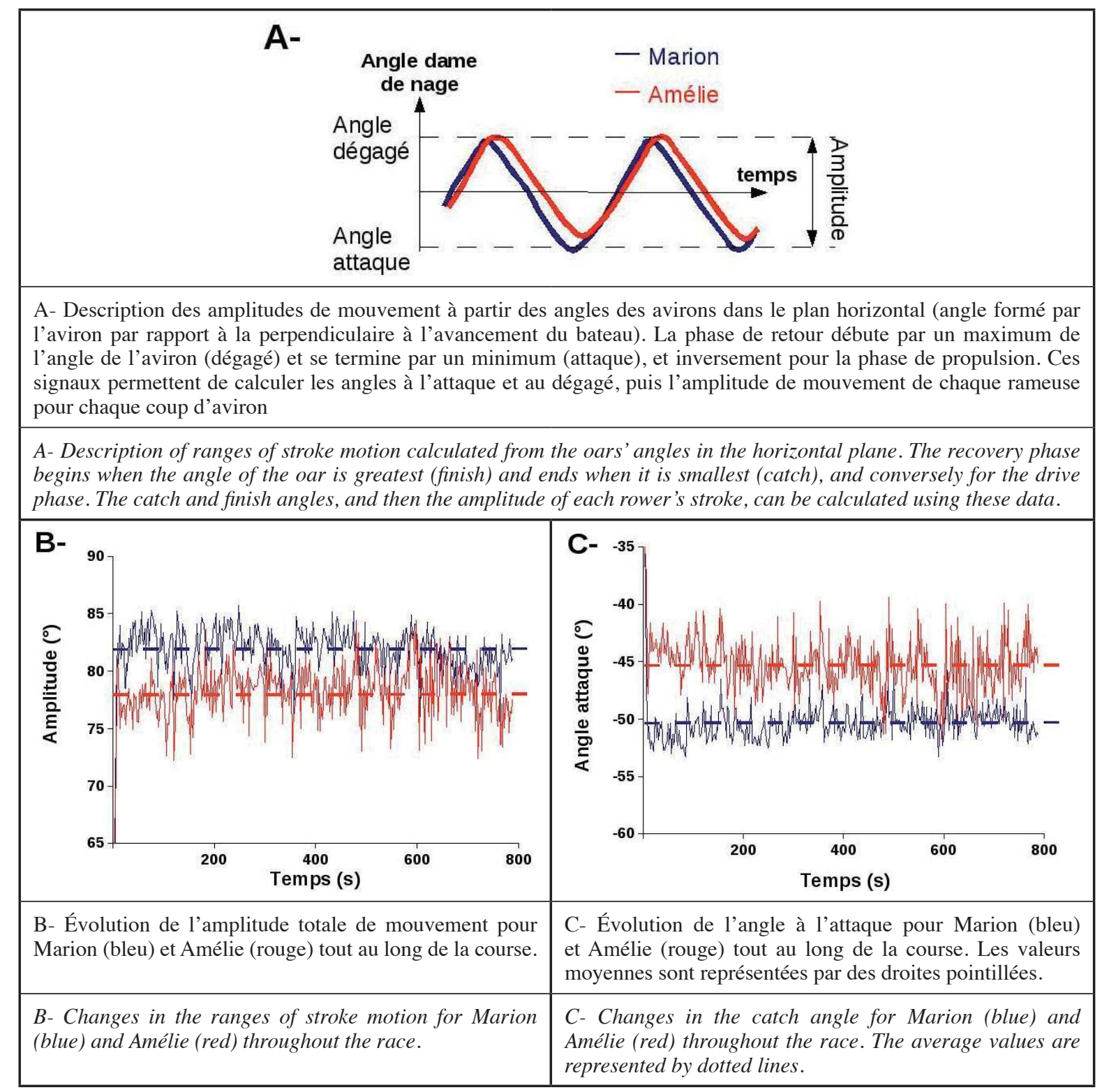

Figure 3 : Test de la deuxième hypothèse

Figure 3: Testing the second hypothesis.

L'analyse des différences de vitesse sur le retour aérien (Figure 4) du mouvement d'aviron entre les deux rameuses a mis en évidence deux phénomènes pouvant être mis en relation avec la perception de Marion «d'être poussée » par Amélie.

Le premier phénomène a été mis en évidence par la comparaison des durées globales des retours des deux rameuses (associé au mouvement de replacement des rameuses vers l'arrière du bateau avant une nouvelle phase propulsive). La Figure 3 montre que les durées de retour étaient parfaitement identiques pour les deux rameuses (différence de durées de retour: $0.00 \pm 0.04 \mathrm{~s}$, Figure 4-A), celles-ci apparaissant comme un élément de référence invariable au sein de l'équipage, expliquant notamment que les deux rameuses ramaient à la même cadence. Ce résultat est compatible avec la perception de Marion: la similitude des durées de retour des deux rameuses implique que Marion, qui avait une amplitude de mouvement plus importante que celle d'Amélie, devait donc se déplacer plus vite lors de son mouvement de replacement sur la coulisse (différence de vitesse : $2.7 \pm 3.5^{\circ} . \mathrm{s}^{-1}$, Figure 4-B) afin de « rattraper » le mouvement d'Amélie, et être synchrones au moment de l'attaque. 
Le deuxième phénomène a été révélé par la comparaison des vitesses respectives de retour pour les deux segments de début et de fin du retour (celui-ci ayant été préalablement été divisé en deux phases d'amplitude égale). Cette analyse a mis en évidence que la vitesse du début de retour était plus grande pour Amélie que pour Marion (différence de vitesse: $-2.6 \pm 6.2^{\circ} . \mathrm{s}^{-1}$, Figure $4-\mathrm{C}$ ), et qu'à l'inverse, logiquement, la vitesse de la fin du retour était plus grande pour Marion que pour Amélie. Ce résultat peut contribuer à expliquer la perception de Marion: il permet de comprendre que celle-ci pouvait se sentir « poussée » par sa coéquipière au début du retour, sensation qui pouvait être renforcée à la fin du retour car elle devait aller encore plus vite sur la fin du retour (différence de vitesse : $8.0 \pm 7.6^{\circ}$. $\mathrm{s}^{-1}$, Figure 4-D), ce qui était susceptible d'accentuer son sentiment d'urgence.

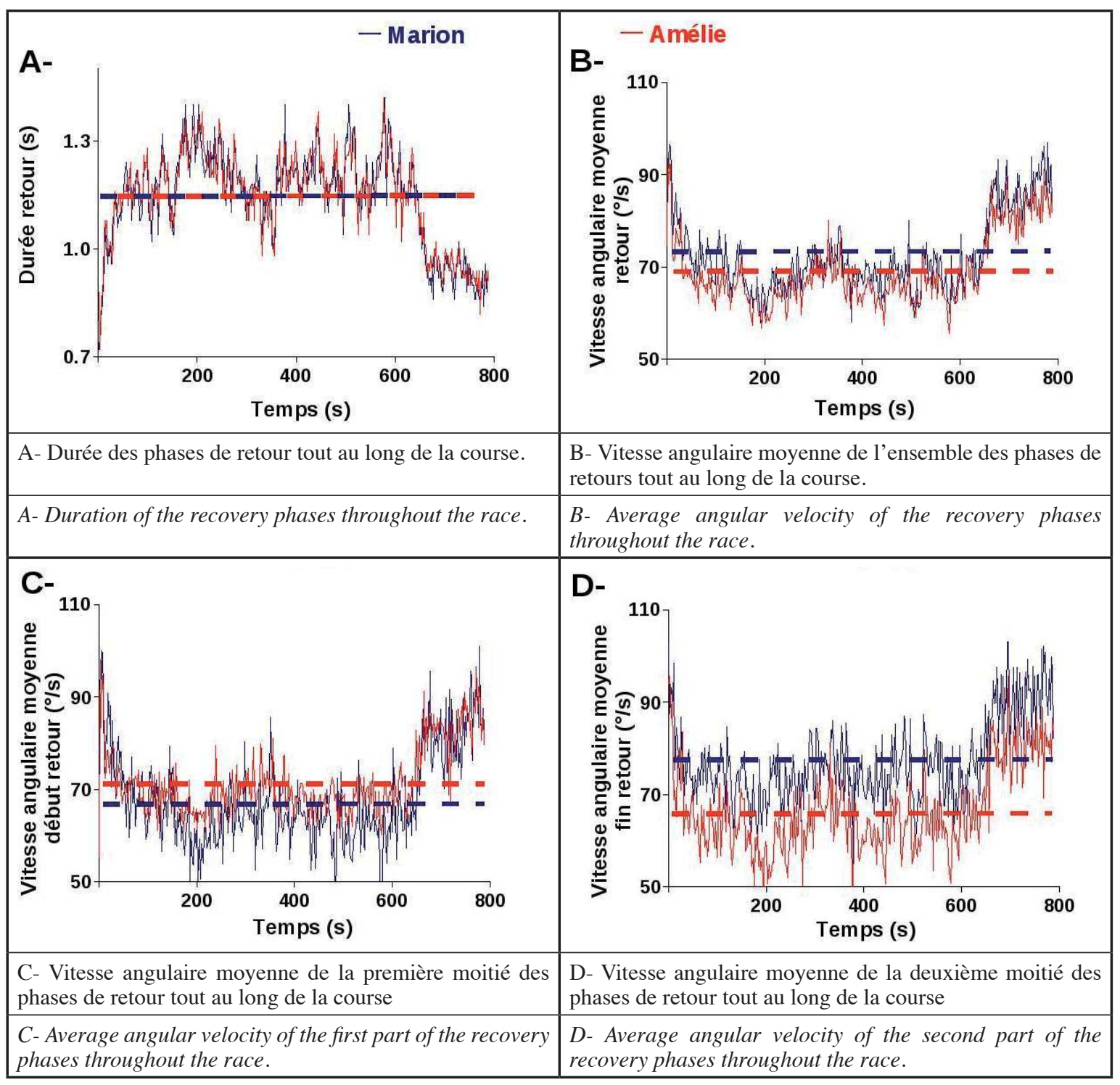

Figure 4 : Test de la troisième hypothèse.

Les valeurs moyennes sont représentées par des droites horizontales pointillées.

Figure 4: Testing the third hypothesis.

The average values are represented by dotted lines.

Ainsi la sensation exprimée par Marion d' «être poussée » par sa coéquipière, et de ne pas pouvoir réaliser pleinement son mouvement sous la pression de sa partenaire s'expliquerait donc principalement par un différentiel d'amplitude entre les deux rameuses. Ce sentiment était probablement amplifié, d'une part, par le manque de stabilité de l'amplitude à l'attaque d'Amélie, et d'autre part, 
par un début de retour plus lent de Marion qui devait donc aller encore plus vite sur la fin du retour pour atteindre son amplitude.

\section{4.- Discussion}

Les résultats de cette étude sont discutés dans trois sections, répondant respectivement à ses visées, (a) empiriques (contribution à la connaissance des coordinations interindividuelles en sport), (b) méthodologiques (fécondité d'une analyse conjointe du cours d'expérience et de paramètres mécaniques), et (c) pratiques (définition d'orientations pour l'entrainement en aviron).

\section{1.- Apports relatifs à la connaissance de la coordination interindividuelle en aviron et plus largement dans les situations sportives}

Les résultats de cette étude mettent en évidence trois caractéristiques de la coordination inter-rameurs qui apportent une contribution originale à la connaissance des conditions de la performance collective en aviron, et plus largement dans les situations sportives ou professionnelles collectives.

\subsection{1.- Le « coût cognitif » de la coordination inter-rameurs}

Cette étude montre que la coordination inter-rameurs n'est pas « réglée à l'avance », mais suppose une activité continue des rameuses dans le cours même des courses, à un niveau de cette activité significative pour les rameuses. Cette activité se traduit par une sensibilité permanente des rameuses à l'état actuel de la synchronisation de leurs mouvements d'aviron (ce qu'elles formulaient de façon syncrétique par la sensation d' " être ensemble » ou de «ne pas être ensemble»), par une exploration et une interprétation de l'activité de leur partenaire, et par des préoccupations d'ajustements mutuels selon des modalités diversifiées. Ainsi la gestion de leur coordination constituait pour les rameuses une part importante de leurs préoccupations, et des éléments sur lesquels elles focalisaient leur attention en course.

Ce résultat interroge les conceptions dominantes relatives à la performance collective en aviron, véhiculées par la littérature technique et professionnelle du domaine, tout autant que par la littérature scientifique. En effet, celle-ci accordent une part prépondérante dans l'explication des performances des équipages, à un ensemble de facteurs relativement stables, et préexistants à l'activité collective elle-même, qu'il s'agisse de variables psycho-sociales (Magyar et al., 2004; Mugford \& Tennant, 2004), de caractéristiques physiologiques (Nilsen, 2005), biomécaniques (Hill, 2002; Wing \& Woodburn, 1995), ou psychologiques (Kellmann, Altenburg, Lormes, \& Steinacker, 2001; Purge, Jurimae, \& Jurimae, 2006) des rameurs, ou encore de leurs « signatures techniques » (Gossé et al., 2008). Cette étude accrédite l'idée que la coordination n'est pas prédéterminée de façon exclusive par ces variables, et qu'elle fait l'objet de processus permanents d'ajustements spécifiques in situ, tout au long de la course, du fait qu'elle soit perpétuellement menacée au cours de celle-ci, par des événements extérieurs imprévus (e.g., passage d'une vague, rafale de vent), ou par la dynamique même de l'activité collective (en particulier par les fluctuations de la perception des rameuses "d'être ensemble » ou non).

On peut ainsi avancer l'hypothèse que la coordination génère un « coût cognitif » pour les rameurs au cours des épreuves, dont la minimisation (ou la meilleure gestion) pourrait être une des caractéristiques de l'expertise des équipages les plus performants. Cette caractéristique incite à considérer l'aviron comme ne dérogeant pas, de ce point de vue - en dépit des contraintes technologiques et mécaniques spécifiques que ce sport impose à l'activité collective - aux phénomènes d'ajustements continus relativement indéterminés, mis en évidence dans d'autres sports collectifs lors de matchs. Bourbousson et al. (2008) ont par exemple montré qu'en basket-ball, la coordination entre les joueurs ne pouvait se réduire à l'application d'un plan prétabli, ou d'un système de jeu, mais que celle-ci était continuellement remise en question par l'activité même des joueurs et par les événements du 
match, conduisant les joueurs à modifier en permanence les coordinations qu'ils entretenaient avec leurs partenaires.

\subsection{2.- Originalité des modes de coordination inter-rameurs}

Nos résultats pointent l'originalité de certains phénomènes relatifs à l'activité des rameuses visant à se coordonner avec leur partenaire, en comparaison de ceux relatés dans les études précédentes en sport. En effet, si l'analyse du cours d'expérience des rameuses a pour partie mis en évidence des phénomènes comparables à ceux qui ont été décrits dans des études antérieures, elle a aussi révélé certaines caractéristiques singulières de l'activité collective des rameuses. Parmi les convergences avec les études précédentes, on peut souligner la prégnance de l'activité d'exploration et d'interprétation de l'activité de son (ou ses) partenaires, permettant à chaque rameuse d'inférer les préoccupations, l'état de fatigue ou les émotions de sa partenaire, ou encore certaines modalités d'ajustements mutuels ( «Inciter sa coéquipière à s'ajuster à soi », et " S'ajuster à sa coéquipière »), qui renvoient à des processus d'enquête et de surveillance, et à des jeux d'influence mutuelle comparables à ceux qui ont été décrits dans les résultats d'études en sport (e.g., Poizat et al., sous presse; Saury, 2008) et dans le travail (e.g., Chauvin \& Lardjane, 2008; Cicourel, 1994; Heath \& Luff, 1992, 1994; Heath, Svensson, Hindmarsh, Luff, \& Vom Lehn, 2002; Hoc, 2003; Salembier \& Zouinar, 2004, 2006; Schmidt, 1994, 2002).

Cependant, au-delà de ces similitudes, l'activité des rameuses révèle des phénomènes particulièrement originaux, que nous résumons en deux points.

Le premier point concerne la sensibilité particulière des rameuses à l'état de la coordination ellemême, qui accompagne de façon continue leur activité, et qu'elles ont exprimé en termes d'expérience d' " être ensemble » ou de « ne pas être ensemble». Certes les études antérieures ont montré que les partenaires d'une même équipe étaient sensibles à un « partage cognitif » avec leur partenaire, ce qui peut également traduire l'idée d'une communauté d'expériences et d'activité (le sentiment d'une intelligibilité partagée d'une situation, d' «être sur la même longueur d'onde »). Cependant, d'une part cette sensibilité apparaissait moins prégnante qu'elle ne l'est en aviron, et d'autre part, elle mettait en jeu des phénomènes tels que le partage d'informations contextuelles (Poizat et al., sous presse), ou le partage d'interprétations ou de jugements relatifs à la situation (Saury, 2008), c'est-à-dire essentiellement des dimensions perceptives et inférentielles de l'expérience. Dans le cas de l'aviron, l'expérience des rameuses d' «être ensemble » ou de " ne pas être ensemble » met en jeu des registres sensoriels variés (associant notamment des sensations corporelles, la perception des comportements du partenaire, des sensations de glisse du bateau, et des jugements du degré de synchronisation des mouvements de rame), traduisant une expérience de « couplage physique », intégrant fortement des dimensions sensori-motrices.

Le deuxième point concerne deux modalités d'ajustements mutuels entre les rameuses, que nous avons nommées « Etre une référence stable pour sa coéquipière », et « Ramer ensemble au service du bateau ». Celles-ci sont particulièrement originales car dans les deux cas, contrairement aux deux autres modalités décrites (i.e., "Inciter sa coéquipière à s'ajuster à soi », et « S'ajuster à sa coéquipière »), elles renvoient pour les rameuses à la préoccupation de se coordonner à sa coéquipière en faisant abstraction de l'activité de cette dernière. Dans le premier cas, il s'agit pour chaque rameuse de se focaliser sur son propre mouvement afin de produire un cycle de rame invariable, et dans le second, il s'agit d'ajuster son activité aux comportements et à la qualité de glisse du bateau. Ces observations renforcent l'idée que la « consistance technique » (i.e., la faible variabilité des coups d'aviron) est, du point de vue des rameurs, une condition cruciale de leur coordination. Elles soulignent aussi l'importance d'une « médiation du bateau » dans la régulation in situ de cette coordination, qui pourrait constituer une spécificité, dans le champ des pratiques sportives ou des pratiques professionnelles, des situations d'activités collectives d'interdépendance forte avec un support mécanique. 


\subsection{3.- Diversité des modalités d'ajustements inter-rameurs}

Nos résultats pointent la diversité des modalités d'ajustements mutuels observés au sein des deux équipages. En effet, celle-ci peut surprendre au regard de la spécialisation fonctionnelle des rôles de « chef de nage » et de « deux » au sein des bateaux d'aviron. Selon la conception classiquement véhiculée dans la littérature technique, cette spécialisation présuppose un mode d'interaction qualifié « d'interaction directe » par Lippens (2005, p. 188), qui consiste à accorder un rôle de « leader » au rameur « chef de nage », celui-ci étant chargé d'impulser une cadence et de fixer les caractéristiques techniques de chaque coup d'aviron (e.g., l'amplitude, la dynamique du mouvement propulsif et du retour), le rameur « deux » étant pour sa part chargé de reproduire la cadence et le mouvement impulsé par le rameur « chef de nage », en minimisant autant que possible les différences (Lippens, 2005).

Nos résultats montrent que, bien que cette organisation fût celle que les rameuses prétendaient vouloir mettre en œuvre, les modalités d'ajustements mutuels entre les rameuses « chefs de nage » et « deux » étaient plus variables. D'une part on observe que, si les préoccupations d' « inciter sa coéquipière à s'ajuster à soi » se manifestaient quasi-exclusivement chez les rameuses « chefs de nage », il était également fréquent que la rameuse « chef de nage » cherche à « S'ajuster à sa coéquipière », contrairement au mode d'organisation défini a priori. D'autre part, les deux modes d'ajustements décrits «Être une référence stable pour sa coéquipière » et «Ramer ensemble au service du bateau » ne renvoient pas à des modes de coordination dans lesquels les rameuses s'ajustent réciproquement l'une à l'autre, mais plutôt à des modes de coordination "médiés » par des effets que les rameuses recherchent respectivement, (a) sur la variabilité de leur propre mouvement d'aviron (qu'elles cherchent à minimiser), et (b), sur la dynamique du comportement global du bateau, en termes de qualité de glisse, de direction ou d'équilibre. Ces observations peuvent être interprétées en relation avec les résultats de Lippens (1999) sur le fonctionnement d'un équipage de « deux de pointe sans barreur » du meilleur niveau mondial. Cet équipage s'organisait, contrairement à la description conventionnelle de la répartition des tâches entre «chef de nage » et « deux », selon un mode que Lippens a qualifié d' ' interaction indirecte »: chaque membre de l'équipage était focalisé sur des attentes liées à son rôle spécifique dans la perspective de contribuer à la meilleure performance du bateau, considéré comme un tout. Une analyse de la variabilité des durées des différentes phases du mouvement d'aviron (propulsive et de retour) pour les rameurs de cet équipage a montré que cette variabilité était plus grande (en particulier lors de la phase propulsive) pour le rameur « chef de nage » que pour le rameur « deux ». Pour Lippens (2005) ce mode de fonctionnement représente une adaptation plus pertinente aux contraintes pesant sur l'activité de l'équipage, que la stratégie d'organisation classique, basée sur une répartition rigide des rôles entre « chef de nage » et « deux ». En effet, elle permet au rameur « chef de nage » d'adapter son mouvement en fonction des réactions du bateau, et en particulier des variations de direction (celui-ci étant le mieux placé pour évaluer les mouvements de poupe du bateau). Au regard de ces analyses, nous pouvons penser que la variabilité des modalités d'ajustements mutuels observés au sein des deux équipages de notre étude peut être due à différents éléments: (a) le niveau d'expertise des athlètes: il s'agissait de rameuses Junior et non d'athlètes au plus haut niveau mondial; (b) la différence de consistance technique (Soper \& Hume, 2004) entre les deux rameuses: contrairement à l'étude de Lippens (1999), la variabilité des mouvements de rame de la rameuse « deux » dans l'équipage 2 était plus importante que celle des mouvements de la rameuse « chef de nage »); et (c) la durée de formation des équipages : il s'agissait dans les deux cas d'équipages de constitution récente, n'ayant pas encore stabilisé de routines collectives.

La variabilité des modalités d'ajustements mutuels observés au sein des équipages d'aviron traduit un phénomène déjà observé dans d'autres situations collectives dans lesquels des rôles spécifiques sont prédéfinis et assignés aux différents membres d'une équipe. En effet, comme cela a été montré en sport (e.g., Bourbousson et al., 2008; Saury, 2008) ou dans le travail (e.g., Cicourel, 1994 ; Munduteguy \& Darses, 2000), ces rôles, tout en en délimitant les contours, ne prescrivent pas totalement les formes d'interaction entre les membres de l'équipe. 


\section{2.- Fécondité d'une analyse conjointe du cours d'expérience et de paramètres mécaniques}

La fécondité de l'analyse que nous avons présentée dans l'étude de cas portant sur l'Equipage 2 est analysée à deux niveaux: (a) la fécondité empirique d'une analyse conjointe du cours d'expérience et de paramètres mécaniques, et (b) les enjeux méthodologiques d'une telle articulation dans le cadre du développement d'un programme « cours d'action » portant sur les situations sportives.

\subsection{1.- Fécondité empirique}

Du point de vue de sa fécondité empirique, l'articulation d'analyses des cours d'expérience des rameuses et de mesures mécaniques, a mis en évidence des caractéristiques de la coordination entre les rameuses, compatibles avec leurs perceptions mais insoupçonnés par elles (et par les entraineurs), ouvrant sur une meilleure compréhension de cette coordination, et sur des pistes d'intervention pour l'entraînement en aviron. En effet, comme nous l'avons souligné dans la section précédente, la reconstruction des cours d'expérience des rameuses en course, en s'attachant à la description de phénomènes de l'activité « significatifs pour elles », a permis des descriptions non triviales de leur activité.

Cependant, cette description se révèle insuffisante pour rendre compte d'autres aspects des coordinations inter-rameurs, qui mettent en jeu de façon déterminante des automatismes et ajustements comportementaux subtils non significatifs pour les rameurs. Par exemple, dans l'étude de cas présentée, les perceptions exprimées par Marion de «ne pas être ensemble », «d'être poussée » par sa coéquipière, et de «ne pas pouvoir réaliser pleinement son mouvement » à certains moments de la Course 2 renvoyaient, à un niveau «significatif pour elle », à des phénomènes syncrétiques de son expérience. S'ils permettaient d'identifier et de localiser un incident critique de son point de vue, ceux-ci ne permettaient pas de comprendre précisément l'origine de ces perceptions dans la dynamique des ajustements comportementaux effectifs entre les rameuses (y compris pour les rameuses elles-mêmes). Or la sensation de la rameuse « chef de nage » « d'être poussée » par sa coéquipière peut être a priori liée à une diversité d'ajustements comportementaux dynamiques entre les rameuses, non seulement non significatifs pour les rameuses, mais également trop subtils pour être identifiables, par observation ou analyse vidéo, par les entraîneurs eux-mêmes. L'analyse de paramètres mécaniques a permis, dans notre étude, d'approfondir la compréhension de la dynamique globale de l'activité collective, en rattachant la perception syncrétique de la rameuse « d'être poussée » par sa coéquipière à une différence d'amplitude des mouvements de rame, et à une différence de vitesse dans le début et la fin de la phase de retour entre les deux rameuses.

Les résultats de cette étude montrent ainsi l'intérêt d'indexer l'analyse mécanique à une analyse préalable du cours d'expérience des rameuses, selon un principe de «primat du cours d'expérience » (Theureau, 2006), cette analyse préalable guidant d'une façon pertinente au regard du vécu subjectif des acteurs le choix des paramètres mécaniques à explorer. Soulignons que cette perspective trouve un certain écho dans la littérature technique et scientifique propre à l'aviron. En effet, Lippens (2005) affirme que l'appréhension de la complexité du mouvement d'aviron suppose de comparer les « données physiques » relatives aux comportements des rameurs avec les « données psychologiques » correspondantes, issues du point de vue des rameurs. Selon cet auteur, cette analyse peut aider les entraîneurs et les rameurs à trouver les relations entre les techniques idéales d'un point de vue biomécanique et les « concepts psychologiques de la technique » des athlètes (p. 185).

\subsection{2.-Fécondité méthodologique}

Du point de vue de ses enjeux méthodologiques, cette étude apporte une contribution exploratoire à l'étude empirique d'un objet théorique encore peu investigué dans le programme « cours d'action »: le « cours d'in-formation ». Dans sa systématisation récente du programme cours d'action, Theureau (2006) a défini différents objets théoriques pour appréhender l'activité humaine, tout en respectant les exigences d'une description qui prenne en compte le caractère asymétrique du couplage structurel 
entre l'acteur et son monde (Theureau, 2006). Cette asymétrie signifie que le domaine de perturbations d'un acteur est défini par cet acteur même : il interagit, à chaque instant, avec un environnement signifiant pour lui, à l'émergence duquel il a lui-même contribué à partir de sa constitution physiologique, de ses caractéristiques psychologiques, de son histoire et de ses propres interactions avec cet environnement à l'instant précédent. Si le cours d'expérience permet d'appréhender les caractéristiques de l'activité et de l'environnement qui sont montrables, racontables et commentables par l'acteur, il laisse dans l'ombre d'autres facettes de l'activité et de l'environnement qui, tout en n'étant ni montrables, racontables et commentables, interviennent dans l'activité et l'expérience de l'acteur.

Theureau a proposé l'objet théorique « cours d'in-formation » pour rendre compte de la dynamique du couplage structurel, ou domaine cognitif de l'acteur (que les éléments qui composent celui-ci soient significatifs ou non pour l'acteur). Le cours d'in-formation intègre des éléments ou événements ressortant du corps, de la situation et de la culture de l'acteur, qui ne sont pas nécessairement « significatifs pour lui » dans le cours de son activité, mais qui sont néanmoins pertinents pour l'organisation interne de l'acteur à chaque instant. La description du cours d'in-formation dépasse donc les limites des descriptions du cours d'expérience de l'acteur dans la mesure où certains éléments n'ayant pas donné lieu à expérience peuvent être pris en compte; elle n'est cependant acceptable, dans une perspective enactive, qu'en accordant un primat au cours d'expérience, autrement dit, en se fondant sur une analyse préalable du cours d'expérience. La notion d'in-formation, empruntée à Varela (1989) (du latin in formare: former de l'intérieur), constitue une alternative, dans le paradigme de l'enaction, à la notion d'information du paradigme cognitiviste. Pour être « acceptable » (c'està-dire, respecter l'asymétrie des interactions entre l'acteur et son environnement), la description du cours d'in-formation passe par celle du cours d'expérience (Theureau, 2006). S'il définit et circonscrit cet objet de manière abstraite, Theureau propose peu de pistes, sur le plan méthodologique, pour documenter empiriquement le cours d'in-formation. Lors d'une étude sur des interactions compétitives (match de simple) et coopératives (match de double) en tennis de table, Poizat (2006) a mis en œuvre une méthodologie articulant une description des cours d'expérience des pongistes et une description fine de leurs comportements observables empruntant à l'ethnographie. Ceci lui a permis de mettre à jour des phénomènes devenus en quelque sorte « transparents » aux pongistes, car fortement routinisés, mais jouant cependant un rôle essentiel dans la construction d'une activité collective. Cette articulation a consisté en un premier pas vers l'objet théorique du cours d'in-formation, en révélant des dimensions de la culture des pongistes incorporées dans leur activité en compétition. La méthodologie présentée dans cette étude nous paraît constituer une contribution supplémentaire à l'étude du cours d'in-formation, en direction de l'exploration de dimensions perceptivo-motrices et auto-adaptatives non significatives pour les acteurs dans le cours de leur activité, mais cependant indissociables de leur cours d'expérience.

\section{3.- Apports à la conception d'aides à l'entraînement en aviron}

Les apports de cette étude à la conception d'aides à l'entraînement en aviron sont envisagés à trois niveaux: (a) un enrichissement de l'analyse de l'activité des rameurs dans une perspective d'optimisation de la performance collective; (b) le développement de ressources réflexives partagées entre les entraîneurs et athlètes; et (c) le développement de l'usage d'un dispositif de mesures mécaniques dans l'entraînement.

\subsection{1.- Optimisation de la performance collective}

La mise en œuvre de cette étude, et les analyses produites qui ont été restituées aux entraîneurs et athlètes dans un délai de quelques semaines après le recueil de données, ont permis d'enrichir l'analyse de l'activité des rameuses d'une façon qui a engendré des adaptations visant à optimiser la performance des deux équipages concernés. Par exemple, l'analyse présentée dans l'étude de cas conduite avec l'Equipage 2 a permis de donner une signification plus précise à la perception de Marion «d'être poussée » par sa coéquipière, ce qui a ouvert sur la définition d'objectifs de travail 
lors des entraînements ultérieurs visant à remédier à ce dysfonctionnement de la coordination de l'équipage. Pour Marion, il s'agissait notamment de transformer la dynamique de son retour en allant plus vite sur le début du retour: l'un des principes d'efficacité technique en aviron demande en effet classiquement aux rameurs de débuter le retour rapidement et de diminuer la vitesse sur la seconde partie du retour afin de limiter les effets d'inertie et la chute de vitesse lors du retour (Hill \& Fahrig, 2009). Pour Amélie, il s'agissait de rechercher une plus grande amplitude de son mouvement de rame, par l'adaptation des réglages du bateau et par une focalisation particulière sur l'augmentation de l'amplitude à l'attaque de son mouvement.

\subsection{2.- Développement de ressources réflexives partagées}

Au-delà des adaptations visant à optimiser la performance des équipages ayant participé à l'étude, l'ensemble des matériaux collectés (enregistrements vidéo et audio des courses, enregistrements des entretiens d'autoconfrontation, mesures mécaniques) et des analyses compréhensives produites « à chaud », systématiquement restituées aux entraîneurs et athlètes, ont permis le développement de ressources réflexives partagées entre les entraîneurs et les athlètes. Ce dispositif d'analyse, encore inédit dans le cadre des pratiques d'entraînement en aviron, a favorisé:

- Une appropriation « déclarative » de leurs expériences par les rameurs grâce aux entretiens d'autoconfrontation, leur permettant d'identifier et de caractériser, de façon plus précise que dans le cadre des débriefings classiques d'entraînements, leur vécu durant les courses;

- Un partage de ces expériences entre les rameuses d'un même équipage, et entre les entraîneurs et les athlètes, leur permettant de confronter leurs points de vue et interprétations relatives au fonctionnement de l'équipage et aux variations de performance, et d'enrichir leur répertoire de ressources partagées;

- La mise en évidence de phénomènes non triviaux et insoupçonnés relatifs à l'activité collective, comme par exemple, outre les modes d'ajustements décrits entre le rameur « chef de nage » et le rameur « deux », le nombre et le contenu de leurs communications verbales durant les courses.

Ainsi le dispositif d'analyse de l'activité des rameurs s'est progressivement intégré, dans le cours de la collaboration entre les acteurs sportifs (entraîneurs et rameuses) comme une composante « réflexive » du dispositif d'entraînement lui-même (Durand, Hauw, Leblanc, Saury, \& Sève, 2005; Sève, Poizat, Saury, \& Durand, 2006).

\subsection{3.- Développement de l'usage d'un dispositif de mesures mécaniques}

La mise en œuvre de cette étude a également contribué au développement de l'usage d'un dispositif de mesures mécaniques, le système Powerline (Peach Innovations), déjà régulièrement utilisé dans l'entraînement en aviron afin de caractériser la performance et constituer des équipages en fonction des «signatures techniques » et des caractéristiques physiques des rameurs (Gossé et al., 2008). L'articulation d'une analyse des cours d'expérience des rameuses et de l'analyse de paramètres mécaniques mesurés à l'aide du dispositif Powerline a permis ce développement dans deux directions.

D'une part, elle a permis de relier des expériences subjectives des rameuses, souvent décrites à l'aide de notions propres à un langage opératif ou de métaphores, aux corrélats mécaniques accompagnant ces expériences. Cela a conduit, notamment, à donner une « signification mécanique » à des sensations décrites par les rameurs de façon syncrétique, comme c'est le cas par exemple lorsqu'elles expriment la sensation d' «être ensemble » ou de «ne pas être ensemble », ou pour reprendre l'exemple donné dans l'étude de cas présentée, lorsqu'une rameuse exprime la sensation d' « être poussée » par sa coéquipière.

D'autre part, cette analyse ouvre sur la possibilité d'une mise en relation plus systématique entre les expériences vécues par les rameurs, notamment leurs jugements relatifs à la coordination de l'équipage ou à la performance globale du bateau, et les mesures « objectives » rendant compte de ces effets extrinsèques. Une telle analyse constitue ainsi une aide pour les athlètes dans la compréhension 
et l'évaluation de la « fiabilité » de leurs propres sensations, et ouvre vers de nouvelles pistes d'entraînement (e.g., identifier les sensations récurrentes lors d'une vitesse de bateau élevée).

\section{5.- Conclusion et perspectives}

Les résultats de cette étude exploratoire, inédite dans le domaine de l'entraînement en aviron, accréditent l'idée que cette discipline sportive offre un ensemble de situations d'études privilégiés pour l'étude de coordinations interindividuelles dans des situations dans lesquelles l'activité collective entretient elle-même des relations d'interdépendance étroite avec un système mécanique (caractéristique qui se retrouve dans diverses autres situations sportives ou professionnelles, qui exigent par exemple le co-pilotage interactif de machines ou d'engins motorisés). Dans le cas de cette étude, les spécificités du « deux de pointe sans barreur » imposent des contraintes particulièrement fortes sur la coordination entre les rameurs car compte-tenu du caractère asymétrique des forces exercées par les rameurs sur le bateau (du fait qu'ils ne disposent que d'un seul aviron), et de leurs différentes positions dans le bateau, la direction et la vitesse du bateau sont directement subordonnées à cette coordination. Il reste à confirmer que les phénomènes observés peuvent être généralisés à l'ensemble des spécialités d'aviron. En effet, par la combinaison de conditions matérielles et réglementaires variées, ces spécialités génèrent de multiples configurations de réalisation de performances collectives par les rameurs. Elles offrent de ce fait des conditions quasi-expérimentales pour favoriser une meilleure compréhension des éléments concourant à une optimisation de la performance collective dans ce genre de situations sportives.

Cette étude a également montré la fécondité empirique et méthodologique d'une articulation entre une analyse des cours d'expérience et une analyse de paramètres mécaniques concomitants. Cette approche nous semble de nature à mieux caractériser les modalités de coordination, au niveau cognitif et sensori-moteur, entre des individus engagés dans la réalisation d'une tâche collective (sportive ou professionnelle) médiée par un support technique. Il s'agit d'identifier, selon la nature de la tâche à réaliser et de la médiation technique support de la coordination, des paramètres pertinents susceptibles d'enrichir la compréhension des phénomènes d'ajustements mutuels entre les acteurs afin de concevoir des dispositifs d'aide à la performance collective.

\section{REMERCIEMENTS}

Cette étude participe à un programme de recherche plus large intitulé « Optimisation de la performance et interactions Homme-Machine en sport automobile et en aviron » (identifié selon l'acronyme « OPERF2A »), financé sur trois ans (2008-2010) dans le cadre de l'appel à projets recherche 2007 de la Région des Pays de la Loire (volet « Paris scientifiques régionaux et spécialités scientifiques »). Les auteurs remercient les entraîneurs et rameurs du Pôle France et Espoir de Nantes pour leur collaboration à ce projet.

\section{RÉFÉRENCES}

Barré S., \& Gossé C. (2007). Présentation d'un outil d'analyse et d'aide à l'entraînement utilisé par les équipes de France d'aviron. Cahiers de l'ENV, 10, 41-47.

Baudouin, A., \& Hawkins, D. (2004). Investigation of biomechanical factors affecting rowing performance. Journal of Biomechanics, 37(7), 969-976.

Bourbousson, J., Poizat, G., Saury, J., \& Sève, C. (2008). Caractérisation des modes de coordination interpersonnelle au sein d'une équipe de basket-ball. @ ctivités, 5(1), 21-39.

Bourbousson, J., \& Sève, C. (sous presse). Construction/déconstruction du référentiel commun d'une équipe de basketball au cours d'un match. Impulsions.

Cicourel, A. V. (1994). La connaissance distribuée dans le diagnostic médical. Sociologie du Travail, 36, 427449. 
Chauvin, C., \& Lardjane, S. (2008). Decision making and strategies in an interaction situation: Collision avoidance at sea. Transportation Research Part F: Psychology and Behaviour, 11(4), 259-269.

Cooke, N. J., Stout, R. J., \& Salas, E. (2001). A knowledge elicitation approach to the measurement of team situation awareness. In M. McNeese, M. Endsley, \& E. Salas (Eds.), New trends in cooperative activities: System dynamics in complex settings (pp 114-139). Santa Monica, CA: Human Factors.

Durand, M., Hauw, D., Leblanc, S., Saury, J., \& Sève, C. (2005). Analyse de pratiques et entraînement en sport de haut niveau. Education Permanente, 161, 54-68.

Eccles, D. W., \& Johnson, M. B. (2009). Letting the social and cognitive merge: New concepts for an understanding of group functioning in sport. In S. D. Mellalieu, \& S. Hanton (Eds.), Applied sport psychology advances: A review (pp 281-316). London: Routledge.

Eccles, D. W., \& Tenenbaum, G. (2004). Why an expert team is more than a team of experts: a socialcognitive conceptualization of team coordination and communication in sport. Journal of Sport and Exercise Psychology, 26, 542-560.

Eccles, D. W., \& Tenenbaum, G. (2007). A social cognitive perspective on team functioning in sport. In G. Tenenbaum \& R. C. Eklund (Eds.), Handbook of sport psychology (pp. 264-283). New York: Wiley.

Fiore, S. M., \& Salas, E. (Eds.) (2006). Team Cognition and expert teams: Emerging insights into learning and performance for exceptional teams [Special issue]. International Journal of Sports and Exercise Psychology, 4.

Gossé, C., Carpentier, C., \& Avanzini, G. (2008). La composition d'équipage en aviron. Les Cahiers de l'entraîneur, 4, 6-13.

Heath, C., \& Luff, P. (1992). Collaboration and control: Crisis management and multimedia technology in London Underground line control rooms. Computer Supported Cooperative Work, 1, 69-94.

Heath, C., \& Luff, P. (1994). Activité distribuée et organisation de l'interaction. Sociologie du Travail, 36, 523-545.

Heath, C., Svensson, M. S., Hindmarsh, J., Luff, P., \& Vom Lehn, D. (2002). Configuring awareness. Computer Supported Cooperative Work, 11, 317-347.

Hill, H. (2002). Dynamics of coordination within elite rowing crews: evidence from force pattern analysis. Journal of Sports Sciences, 20(2), 101-117.

Hill, H., \& Fahrig, S. (2009). The impact of fluctuations in boat velocity during the rowing cycle on race time. Scandinavian Journal of Medicine \& Science in Sports, 19(4), 585-594.

Hoc, J.-M. (2003). Coopération humaine et systèmes coopératifs. In G. Boyd (Ed.), Ingénierie cognitive, IHM et cognition (pp 139-187). Paris: Hermès.

Hofmijster M. J., Landman E. H., Smith R. M., \& Van Soest A. J. (2007). Effect of stroke rate on the distribution of net mechanical power in rowing. Journal of Sports Sciences, 25, 403-411.

Kellmann, M., Altenburg, D., Lormes, W., \& Steinacker, J. M. (2001). Assessing stress and recovery during preparation for the world championships in rowing. The Sport Psychologist, 15, 151-167.

Lenk, H. (1969). Top performance despite internal conflict: An antithesis to a functionalistic proposition. In J. W. Loy \& G. S. Kenyon (Eds.), Sport, culture and society: A reader on the sociology of sport (pp 393 396). Toronto: Collier-Macmillan.

Lippens, V. (1999). The temporal and dynamic synchronization of movement in coxless oared shells. In P. Blaser (Ed.), Sport kinetics 1997: Theories of motor performance and their reflections in practice (vol. 2) (pp 39-44). Hamburg: Czwalina.

Lippens, V. (2005). Inside the rower's mind. In V. Nolte (Ed.), Rowing faster (pp 185-194). Champaign, IL: Human Kinetic.

Magyar, T. M., Feltz, D. L., \& Simpson, I. P. (2004). Individual and crew level determinants of collective efficacy in rowing. Journal of Sports \& Exercise Psychology, 26, 136-153. 
Mugford, A. L., \& Tennant, L. K. (2004). An investigation of the relationship between cohesion and flow state in varsity and novice rowers. Journal of Sport \& Exercise Psychology, 26(Suppl.), 139.

Munduteguy, C., \& Darses, F. (2000). Facteurs de transgression d'un mode de coopération prescrit pour un mode de coopération adapté au problème à résoudre. In T. H. Benchekroun, \& A. Weill-Fassina (Eds.), Le travail collectif. Perspectives actuelles en ergonomie (pp. 165-190). Toulouse: Octarès.

Nilsen, T. (2005). Selecting athletes and crews. In V. Nolte (Ed.), Rowing faster (pp 229-236). Champaign, IL: Human Kinetics.

Nolte, V. (Ed.) (2005). Rowing faster. Champaign, IL: Human Kinetics.

Poizat, G. (2006). Analyse en ergonomie cognitive de l'activité collective en tennis de table. Contribution à la connaissance des interactions humaines. Thèse de Doctorat en STAPS non publiée de l'Université de Rouen.

Poizat, G., Bourbousson, J., Saury, J., \& Sève, C. (sous presse). Analysis of contextual information sharing during table tennis matches: An empirical study on coordination in sports. International Journal of Sport and Exercise Psychology.

Poizat, G., Sève, C., \& Saury, J. (2007, Septembre). Intelligibilité mutuelle et construction de signification dans les interactions homme-homme: un exemple en tennis de table. Communication présentée au Congrès EPIQUE'07, Nantes (Session thématique : «L'intelligibilité mutuelle dans la coopération homme-homme et la coopération homme-machine »).

Poizat, G., Sève, C., Serres, G., \& Saury, J. (2008). Analyse du partage d'informations contextuelles dans deux formes d'interaction sportives : coopérative et concurrentielle. Le Travail Humain, 71, 323-357.

Purge, P., Jurimae, J., \& Jurimae, T. (2006). Hormonal and psychological adaptation male rowers during prolonged training. Journal of Sports Sciences, 24(10), 1075-1082.

Reimer, T., Park, E. S., \& Hinsz, V. B. (2006). Shared and coordinated cognition in competitive and dynamic task environments: An information-processing perspective for team sports. International Journal of Sport and Exercise Psychology, 4, 376-400.

Salembier, P., \& Zouinar, M. (2004). Intelligibilité mutuelle et contexte partagé. Inspirations théoriques et réductions technologiques. @ctivités, 1,64-85.

Salembier, P., \& Zouinar, M. (2006). Pas de coopération sans partage! Le partage d'information comme régulateur de la cognition individuelle et collective. In F. Jeffroy, J. Theureau, \& Y. Haradji (Eds.), Relation entre activité individuelle et activité collective: Confrontation des différentes démarches d'études (pp. 55-75). Toulouse: Octarès.

Saury, J. (2008). La coopération dans les situations d'intervention, de performance et d'apprentissage en contexte sportif. Contribution au développement d'un programme de recherche en ergonomie cognitive des situations sportives en STAPS. Note de synthèse d'Habilitation à Diriger des Recherches, Université de Nantes.

Schmidt, K. (1994). Cooperative work and its articulation : requirements for computer support. Le Travail Humain, 57, 345-366.

Schmidt, K. (2002). The problem with 'awareness'. Computer Supported Cooperative Work, 11, 285-298.

Sève, C., Bourbousson, J., Poizat, G., \& Saury, J. (2010). Cognition et performance collectives en sport. Intellectica, 52(2), 71-95.

Sève, C., Poizat, G., Saury, J., \& Durand, M (2006). Un programme de recherche articulant analyse de l'activité en situation et conception d'aides à la performance : un exemple en entraînement sportif de haut niveau.@ctivités,3(2),46-64.

Soper, C., \& Hume, P. A. (2004). Towards an ideal rowing technique for performance: the contributions from biomechanics. Sports Medicine, 34(12), 825-848.

Theureau, J. (2004). Le cours d'action: méthode élémentaire. Toulouse: Octarès.

Theureau, J. (2006). Le cours d'action: méthode développée. Toulouse: Octarès. 
Varela, F. J. (1989). Autonomie et connaissance. Paris: Seuil.

Wing, A. M., \& Woodburn, C. (1995). The coordination and consitency of rowers in a rowing eight. Journal of Sports Sciences, 13, 187-197.

\section{RÉsumé}

Cette étude visait à analyser les phénomènes associés à la coordination entre les rameurs d'un équipage au cours d'une course d'aviron, en articulant une analyse des cours d'expérience des rameurs, et une analyse de paramètres mécaniques corrélatifs de leur activité durant la course. Elle a été conçue afin (a) d'améliorer la compréhension de la performance collective et d'optimiser l'entraînement en aviron, et (b) d'explorer la fécondité d'une méthode permettant de décrire les processus de coordination conjointement aux niveaux significatif et non significatif de leur activité pour les acteurs. Deux équipages féminins ont volontairement participé à cette étude. Leur activité a été étudiée in situ lors de deux courses contre la montre, en référence au cadre théorique et méthodologique du cours d'action. Des mesures mécaniques ont conjointement été collectées, permettant de calculer un ensemble de paramètres mécaniques relatifs aux performances des rameurs et à leur coordination. Les résultats présentent, dans la première partie, les phénomènes significatifs pour les rameuses liés à leur coordination. Trois phénomènes ont été mis en évidence: (a) une sensibilité particulière des rameuses à l'état de la coordination, (b) une interprétation récurrente de l'activité de leur partenaire, et (c) quatre modalités typiques d'ajustements mutuels. Dans la deuxième partie, une étude de cas présente l'analyse d'un moment d'une course vécu par les rameuses comme un dysfonctionnement de leur coordination, sur la base de l'articulation d'une analyse des cours d'expérience et de paramètres mécaniques. Ces résultats sont discutés à trois niveaux, répondant aux visées empiriques, méthodologiques et pratiques de l'étude.

MotS CLÉS Sport, Performance collective, Aviron, Coordination Interindividuelle, Cours d'expérience, Cours d'in-formation

\section{RÉFÉRENCEMENT}

Saury, J., Nordez, A., \& Sève, C. (2010). Coordination interindividuelle et performance en aviron: apports d'une analyse conjointe du cours d'expérience des rameurs et de paramètres mécaniques. Activités, 7(1), pp. 2-27. http://www.activites.org/v7n1/v7n1.pdf

Article soumis le 2 septembre 2009, accepté pour publication le 16 novembre 2009. 